\title{
Efficient generation of functional haploid spermatids from human germline stem cells by three-dimensional-induced system
}

\author{
Min Sun ${ }^{1} \cdot$ Qingqing Yuan ${ }^{1} \cdot$ Minghui Niu ${ }^{1} \cdot$ Hong Wang ${ }^{1} \cdot$ Liping Wen $^{1} \cdot$ Chencheng Yao $^{1} \cdot$ Jingmei Hou $^{1}$. \\ Zheng Chen ${ }^{1} \cdot$ Hongyong $\mathrm{Fu}^{1} \cdot$ Fan $\mathrm{Zhou}^{1} \cdot \mathrm{Chong} \mathrm{Li}^{2} \cdot$ Shaorong Gao ${ }^{2} \cdot$ Wei-Qiang Gao ${ }^{1} \cdot{\mathrm{Zheng} \mathrm{Li}^{3}}^{3}$. \\ Zuping $\mathrm{He}^{1,4,5,6}$
}

Received: 21 August 2017 / Revised: 2 October 2017 / Accepted: 17 October 2017 / Published online: 5 January 2018

(c) The Author(s) 2018. This article is published with open access

\begin{abstract}
Generation of functional spermatids from human spermatogonial stem cells (SSCs) in vitro is of utmost importance for uncovering mechanisms underlying human germ cell development and treating infertility. Here we report a threedimensional-induced (3D-I) system by which human SSCs were efficiently differentiated into functional haploid spermatids. Human SSCs were isolated and identified phenotypically. Meiotic chromatin spreads and DNA content assays revealed that spermatocytes and haploid cells were effectively generated from human SSCs by 3D-I system. Haploid cells derived from human SSCs harbored normal chromosomes and excluded Y chromosome microdeletions. RNA sequencing and bisulfite sequencing analyses reflected similarities in global gene profiles and DNA methylation in human SSCs-derived spermatids and normal round spermatids. Significantly, haploid spermatids generated from human SSCs via 3D-I system were capable of fertilizing mouse oocytes, which subsequently enabled the development of hybrid embryos. This study thus provides invaluable human male gametes for treating male infertility.
\end{abstract}

Edited by E. Baehrecke

Electronic supplementary material The online version of this article (https://doi.org/10.1038/s41418-017-0015-1) contains supplementary material, which is available to authorized users.

Zuping He

zupinghe@sjtu.edu.cn

1 State Key Laboratory of Oncogenes and Related Genes, RenjiMed X Clinical Stem Cell Research Center, Ren Ji Hospital, School of Medicine, Shanghai Jiao Tong University, 160 Pujian Road, Shanghai 200127, China

2 Clinical and Translational Research Center of Shanghai First Maternity \& Infant Hospital, School of Life Sciences and Technology, Tongji University, Shanghai 200092, China

3 Department of Andrology, Urologic Medical Center, Shanghai General Hospital, Shanghai Jiao Tong University, 100 Haining Road, Shanghai 200080, China

4 School of Medicine, Hunan Normal University, 371 Tongzhi Road, Changsha, Hunan 410013, China

5 Shanghai Key Laboratory of Assisted Reproduction and Reproductive Genetics, Shanghai 200127, China

6 Shanghai Key Laboratory of Reproductive Medicine, Shanghai 200025, China

\section{Introduction}

Infertility has currently become one of the most serious issues affecting human reproduction and health, due to genetic variants and segregating alleles [1, 2], environmental pollution, and epigenetic factors. It has been reported that about $15 \%$ of couples are infertile in the USA [3], and half of them result from male infertility [4]. In China, it has been estimated that 50 million of men are infertile, and notably, azoospermia comprise $25 \%$ of male infertility cases [5]. Therefore, it is of great interest to generate functional gametes for patients with male infertility especially for azoospermia. Spermatogenesis is a complex process by which spermatogonial stem cells (SSCs) self-renew and differentiate into haploid spermatids within the elaborated microenvironment or the niche in the seminiferous epithelium. Any errors that occur during spermatogenesis can lead to male infertility [6]. Owing to the destroyed niche of testes, azoospermic patients with SSCs are usually unable to produce functional spermatids. Thus, generating human male gametes in vitro has retained a key issue and central goal in the field of cell biology and reproductive medicine [3]. Production of spermatids in vitro would not only provide male gametes for azoospermic patients but also offers 
$\mathbf{A}$

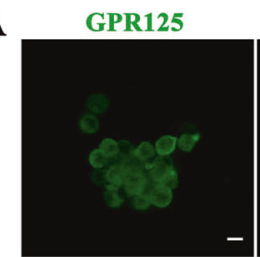

B
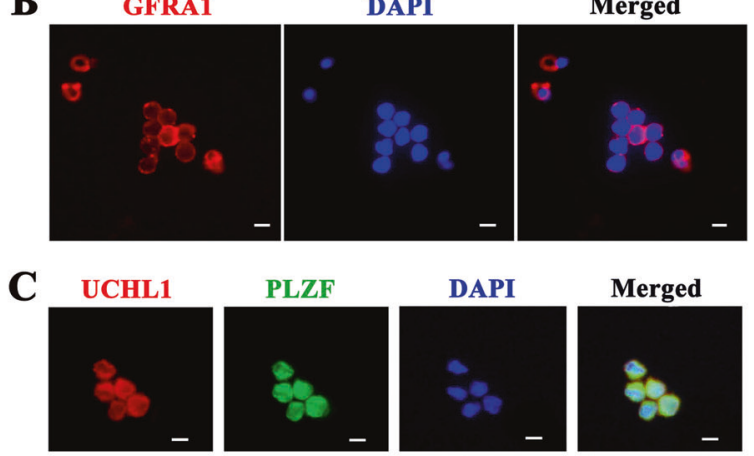

D Rabbit IgG
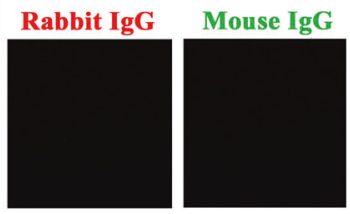

$\mathbf{E}$

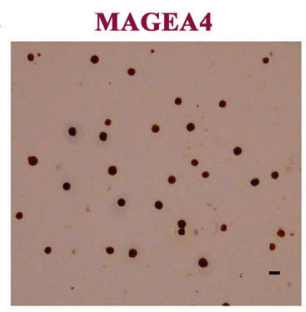

$\mathbf{F}$

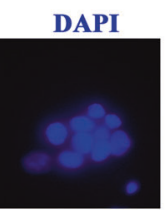

Merged
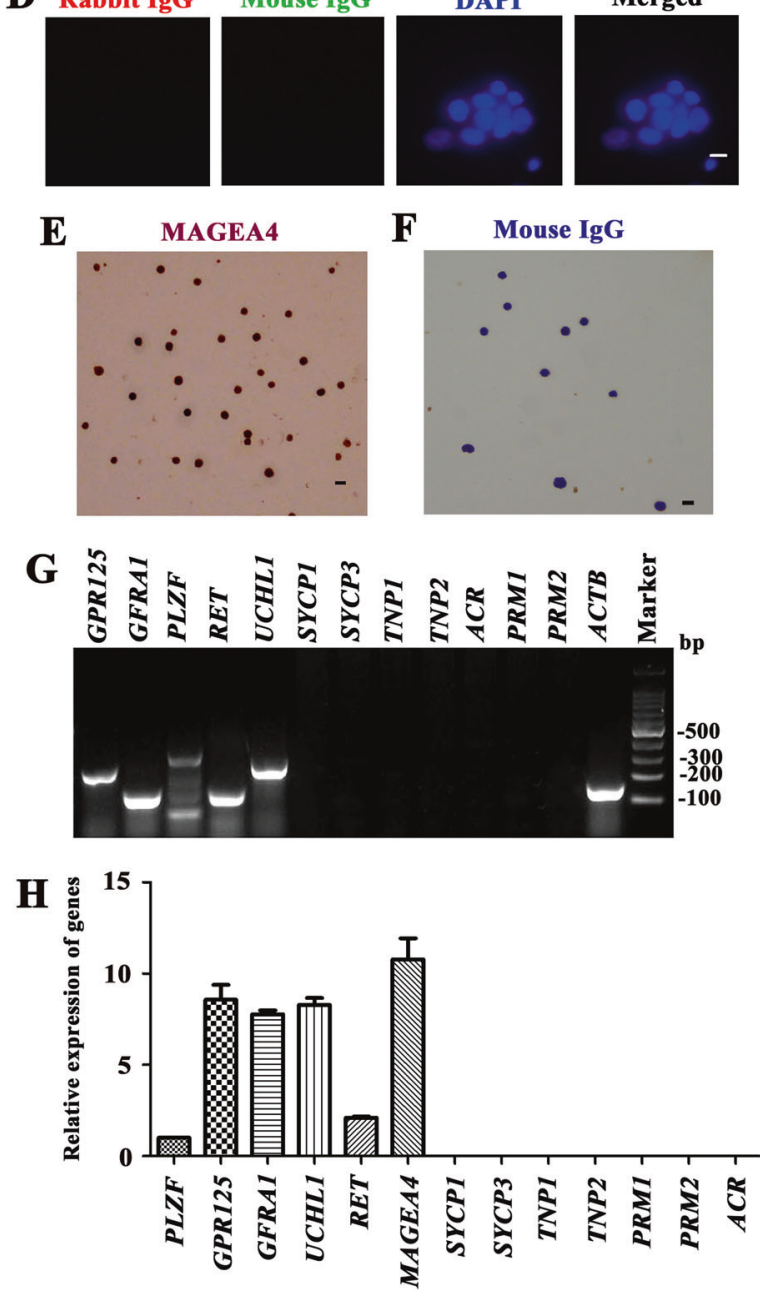

an excellent platform for investigating molecular mechanisms underlying human germ cell development and male infertility [7].
Fig. 1 Identification of human SSCs. a-f Immunocytochemistry revealed the expression of GPR125 (a), GFRA1 (b), UCHL1 and PLZF (c), and MAGEA4 (e) in human GPR $125^{+}$spermatogonia by MACS. Replacement of primary antibodies with isotype IgGs $\mathbf{d}, \mathbf{f}$ were utilized as negative controls. Scale bars in $\mathbf{a}-\mathbf{f}=10 \mu \mathrm{m}$. The data shown in $\mathbf{a}-\mathbf{f}$ were presented from three independent experiments. $\mathbf{g}$ RT-PCR showed the transcripts of GPR125, GFRA1, PLZF, UCHL1, RET, SYCP1, SYCP3, TNP1, ACR, PRM1, and PRM2 in the isolated human GPR $125^{+}$spermatogonia. $A C T B$ was used as a loading control of total RNA. h Real -time PCR revealed the relative expression levels of PLZF, GPR125, GFRA1, UCHL1, RET, MAGEA4, SYCP1, SYCP3, TNP1, ACR, PRM1, and PRM2 to the housekeeping gene $A C T B$ in the isolated human GPR $125^{+}$spermatogonia. The results shown in $\mathbf{g}$ and $\mathbf{h}$ were presented from three independent experiments

Various methods for the in vitro derivation of male germ cells have been developed, mostly based on the twodimensional (2D) culture, the implementation of defined medium, and feeder cells [8-10]. These studies illustrate that spermatogenesis including meiosis can be initiated in vitro. However, the 2D culture cannot effectively mimic the microenvironment of testis due to the lack of relevant growth factors and the disruption of spatial structure, and thus its differentiation efficiency is relatively low. In contrast to conventional 2D culture, the three-dimensional (3D) culture could provide an ideally spatial environment for the cells. Moreover, the 3D culture technique can build a system in vitro, which is closed to the cell developmental microenvironment in vivo. The 3D culture can be utilized to probe the interactions among male germ cells, somatic cells (e.g., Sertoli cells and Leydig cells), and the extracellular matrix (ECM) during spermatogenesis [11-14]. In addition, the $3 \mathrm{D}$ culture system has been used to generate male germ cells in rodents. It has been shown that $3 \mathrm{D}$ culture using collagen matrix for mouse testicular cells promotes the differentiation of germ cells to spermatids [15]. In addition, testicular tissues of newborn mice and mouse SSCs can be induced to differentiate into functional spermatids in vitro using 3D culture system [16-18], but the efficiency is exceedingly low, usually $<2 \%$. Round spermatids can be derived in vitro from mouse spermatogonia [19]; nevertheless, the functionality of the spermatids has not been evaluated. Recently, mouse embryonic stem (ES) cells can be differentiated to germ cells, which can complete meiosis in vitro and give rise to spermatids with fertilization and healthy offspring [20]. However, generation of functional spermatids in vitro has not yet been achieved in humans but is highly anticipated. In the present study, we have for the first time reported the detailed information on a 3D-induced (3D-I) system as well as molecular and cellular evidence demonstrating efficient differentiation of human SSCs into functional spermatids in vitro. According to the "gold standard" criteria for the in vitro-derived gametes [3], phenotypic characteristics, DNA content, chromosome content, 
chromosomal synapsis and recombination, Y chromosome microdeletions, genetic and epigenetic imprinting, and fertilization and development capacity were detected in the resulting haploid cells derived from human SSCs by our 3D-I system. Of unusual significance, our ability of generating human functional haploid spermatids from human SSCs offers an invaluable source of functional male gametes for treating infertility of azoospermic patients. This study could also provide a basis to explore mechanisms underlying the development of human germ cells and etiology of male infertility.

\section{Results}

\section{Isolation and identification of human SSCs and Sertoli cells}

We first isolated GPR125-positive spermatogonia from testicular tissues of obstructive azoospermia (OA) patients using a two-step enzymatic digestion followed by magneticactivated cell sorting (MACS) (Supplementary Fig. S1). Normal spermatogenesis was observed in the testis of OA patients (Supplementary Fig. S2A). Human seminiferous tubules (Supplementary Fig. S2B) were isolated from the testis tissues using the first enzymatic digestion and extensive washes using phosphate-buffered saline (PBS) to remove potential contamination of myoid cells and Leydig cells. Leydig cells secrete testosterone that regulates the progression of meiosis, and thus testosterone was used as an alternative for Leydig cells in the 3D-I system. Male germ cells and Sertoli cells (Supplementary Fig. S2C) were obtained using a second enzymatic digestion, and they were cultured with DMEM/F-12 and $10 \%$ fetal bovine serum (FBS) overnight for differential plating. Human GPR125positive spermatogonia (Supplementary Fig. S2D) were separated by MACS using an antibody against GPR125. The viability of freshly isolated cells was over $98 \%$, as assessed by trypan blue exclusion (data not shown). To assess the identity of the isolated cells, immunocytochemistry revealed that more than $95 \%$ of the freshly isolated cells were positive for GPR125 (Fig. 1a), GFRA1 (Fig. 1b), UCHL1 and PLZF (Fig. 1c), and MAGEA4 (Melanoma antigen family A4) (Fig. 1e), hallmarks for human spermatogonia and SSCs [21]. Replacement of primary antibodies with isotype IgGs served as negative controls, and no immunostaining was seen in the freshly isolated cells (Fig. 1d, f), thus verifying specific expression of the proteins mentioned above in these cells. Reverse transcription (RT)-PCR and real-time PCR further showed that GPR125positive cells expressed the transcripts of GPR125, GFRA1, PLZF, UCHL1, MAGEA4, and RET (Fig. 1g, h). In contrast, mRNA of SYCPI (synaptonemal complex protein 1),
SYCP3 (synaptonemal complex protein 3), specific markers for meiotic germ cells, TNP1 (transition protein 1), TNP2 (transition protein 2), ACR (acrosin), PRMI (protamine 1), and PRM2 (protamine 2), markers for spermatids [22], was undetectable in these cells (Fig. 1g, h), thus excluding the contamination of haploid cells from the testes. Collectively, these data suggest that the freshly isolated cells by MACS using anti-GPR125 are human SSCs phenotypically.

Human Sertoli cells were separated from OA patients using a two-step enzymatic digestion and differential plating as previously described [21]. RT-PCR showed that SOX9, WT1, SCF, BMP4, GDNF, and FSHR genes (markers for human Sertoli cells) were expressed in the isolated cells (Supplementary Fig. S3A). Conversely, GPR125, UCHL1, PLZF, SYCP1, SYCP3, ACR, and PRM2 genes, hallmarks for male germ cells including spermatogonia, spermatocytes and spermatids, were not observed in these cells (Supplementary Fig. S3A). As a positive control, the transcripts of these genes mentioned above were detected in human testis of OA patients (Supplementary Fig. S3B). Immunocytochemistry further revealed that SOX9 (Supplementary Fig. S3C), WT1 (Supplementary Fig. S3D), SCF (Supplementary Fig. S3E), and BMP4 (Supplementary Fig. S3F) were present in the isolated cells. Replacement of primary antibodies with isotype IgGs, and no immunostaining was seen in the isolated cells (Supplementary Fig. S3G), which confirms specific expression of these proteins described above in these cells. Altogether, these results indicate that the isolated cells are human Sertoli cells in phenotype with a high purity, and thus they were utilized in this study as feeder cells for culturing human SSCs after inactivation with mitomycin C (MMC).

\section{Generation of spermatocytes and haploid spermatids from human SSCs by 3D-I system}

The development of human SSCs into spermatocytes and spermatids by the 3D-I system (Supplementary Fig. S1) was first evaluated morphologically. Human SSCs and Sertoli cells (Fig. 2a, left panel) were cocultured with the defined medium in the 3D matrigel, namely 3D-I system. After 10-15 days of induction, spermatocytes (Fig. 2a, middle panel) with condensed chromatin (inserted picture) and a diameter of $14-16 \mu \mathrm{m}$ were observed, and spermatids (Fig. 2a, right panel) with round shape and a diameter of 5-6 $\mu \mathrm{m}$ occurred at day 20 of culture. Immunocytochemistry in situ displayed that SYCP3- and ACR-positive cells were detected in the culture plates of human SSCs by 3D-I system after induction for 20 days (Fig. 2b), reflecting the presence of spermatocytes and spermatids in the 3D-I system.

We next compared the differentiation potential of human SSCs from OA patients under various kinds of induction 
Fig. 2 Morphological and phenotypic characteristics of cells derived from human SSCs by 3D-I system. a Human SSCs (arrow, left panel) and inactivated Sertoli cells were cocultured in the matrigel with the defined medium. Spermatocytes (arrows, middle panel) with condensed chromatins (inserted picture) emerged in the 3D-I system at day $10-15$. Round spermatids (arrowheads, right panel) were seen in the 3D-I system at day 20 . The data shown in a were presented from at least seven independent experiments. Scale bars $=10$ $\mu \mathrm{m}$. b Immunocytochemistry in situ showed the presence of SYCP3 and ACR proteins in the culture plates of human SSCs by 3D-I at day 20. The data shown in $\mathbf{b}$ were presented from at least three independent experiments. Scale bar $=10 \mu \mathrm{m}$. c Real-time PCR showed the relative expression levels of $S Y C P 1$, SYCP3, ACR, TNP1, TNP2, $P R M 1$, and $P R M 2$ to the housekeeping gene $A C T B$ in the cells derived from human SSCs by 3D-I, 2D-I, 3D-C, 3D-N, and 2D-C at day 15. Notes: Post-hoc test method was used to compare the five groups. * indicated $p<$ 0.05 and $* *$ denoted $p<0.01$. d Real-time PCR displayed the relative expression levels of SYCP1, SYCP3, ACR, TNP1, $T N P 2, P R M 1$, and $P R M 2$ to the housekeeping gene $A C T B$ in the cells derived from human SSCs after inducing for 5 days,

10 days, 15 days, and 20 days in 3D-I system. Notes: * indicated $p<0.05$ and $* *$ denoted $p<$ 0.01 in the cells derived from human SSCs after induction for 10 days, 15 days, 20 days compared to 5 days. The results shown in $\mathbf{c}$ and $\mathbf{d}$ were presented from at least three independent experiments
A
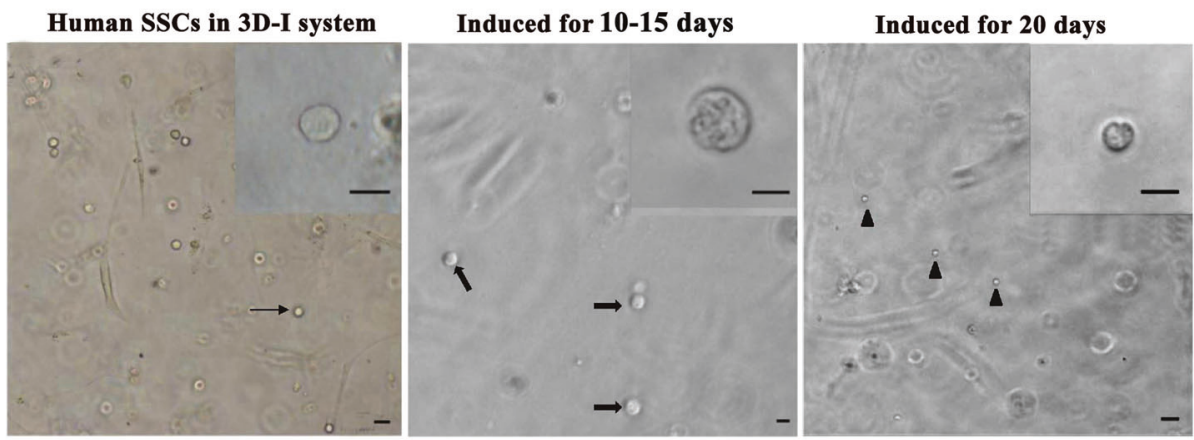

B

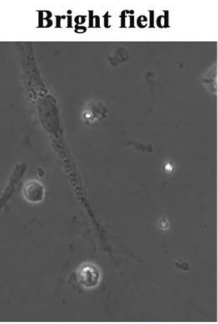

SYCP3

ACR
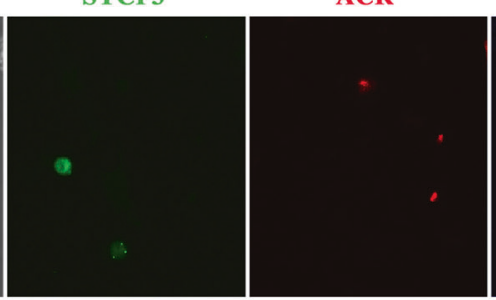

DAPI
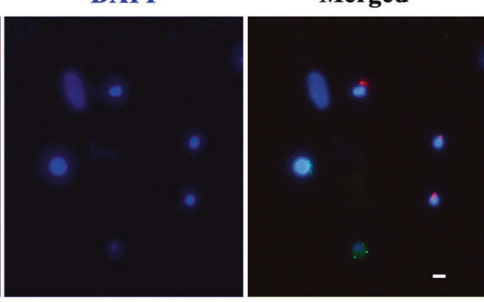

C
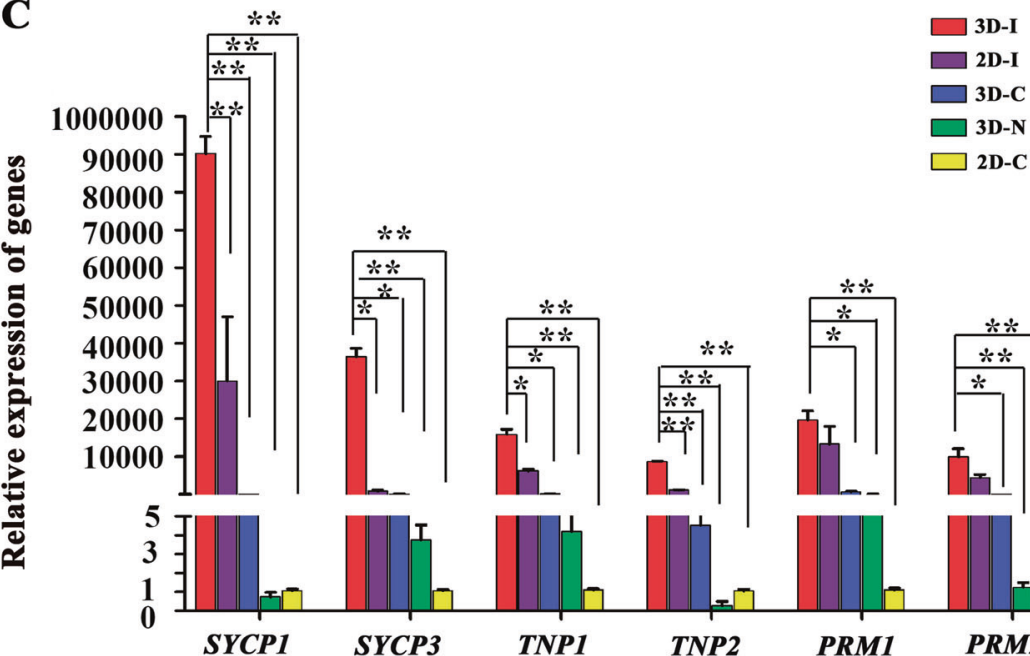

D-I 3D-N 2D-C

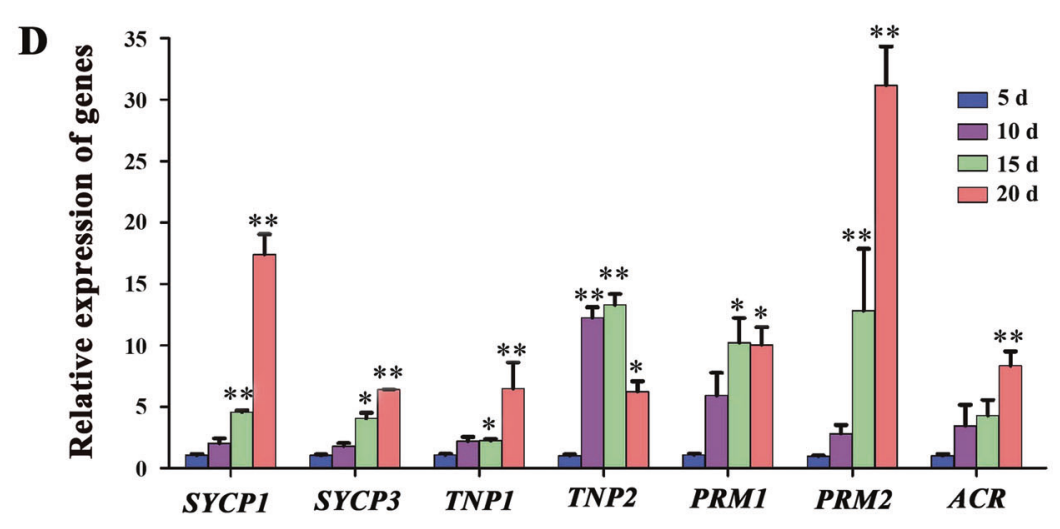

conditions. Real-time PCR revealed that the transcripts of SYCP1 and SYCP3 (hallmarks of meiotic germ cells) as well as ACR, TNP1, TNP2, PRM1 and PRM2 (markers for haploid germ cells) [22], were significantly upregulated in
3D-I group, compared to the 2D-induced (2D-I) group (human SSCs without matrigel but with the defined medium), 3D-control (3D-C) group (human SSCs without Sertoli cells but with matrigel and defined medium), 
Fig. 3 Meiotic progression of the cells derived from human SSCs by 3D-I system. a Meiotic spread assays revealed the coexpression of SYCP3 (red fluorescence), MLH1 (green fluorescence), and CREST (blue fluorescence) in human SSCsderived cells in the 3D-I system after 20 days of culture (upper panel). b The co-expression of SYCP3 (red fluorescence), MLH1 (green fluorescence), and CREST (blue fluorescence) in human pachytene spermatocytes served as a positive control. Scale bars in $\mathbf{a}$ and $\mathbf{b}=10 \mu \mathrm{m}$. c Meiotic spread assays showed cellular localization of SYCP3 (red fluorescence) and $\gamma \mathrm{H} 2 \mathrm{AX}$ (green fluorescence) in human SSCs-derived cells in the 3D-I system after 20 days of induction. d The colocalization of SYCP3 (red fluorescence) and $\gamma \mathrm{H} 2 \mathrm{AX}$ (green fluorescence) in human pachytene spermatocytes was used as a positive control. e Replacement of primary antibodies with isotype IgGs were utilized as negative controls. Scale bars in $\mathbf{c}-\mathbf{e}=10$ $\mu \mathrm{m}$. f Meiotic spread assays showing the percentage of SYCP3-positive cells in 3D-I group, 2D-C group, and 2D-I group. Notes: * indicated $p<$ 0.05 and $* *$ denoted $p<0.01$.

The data shown in a-f were presented from at least three independent experiments
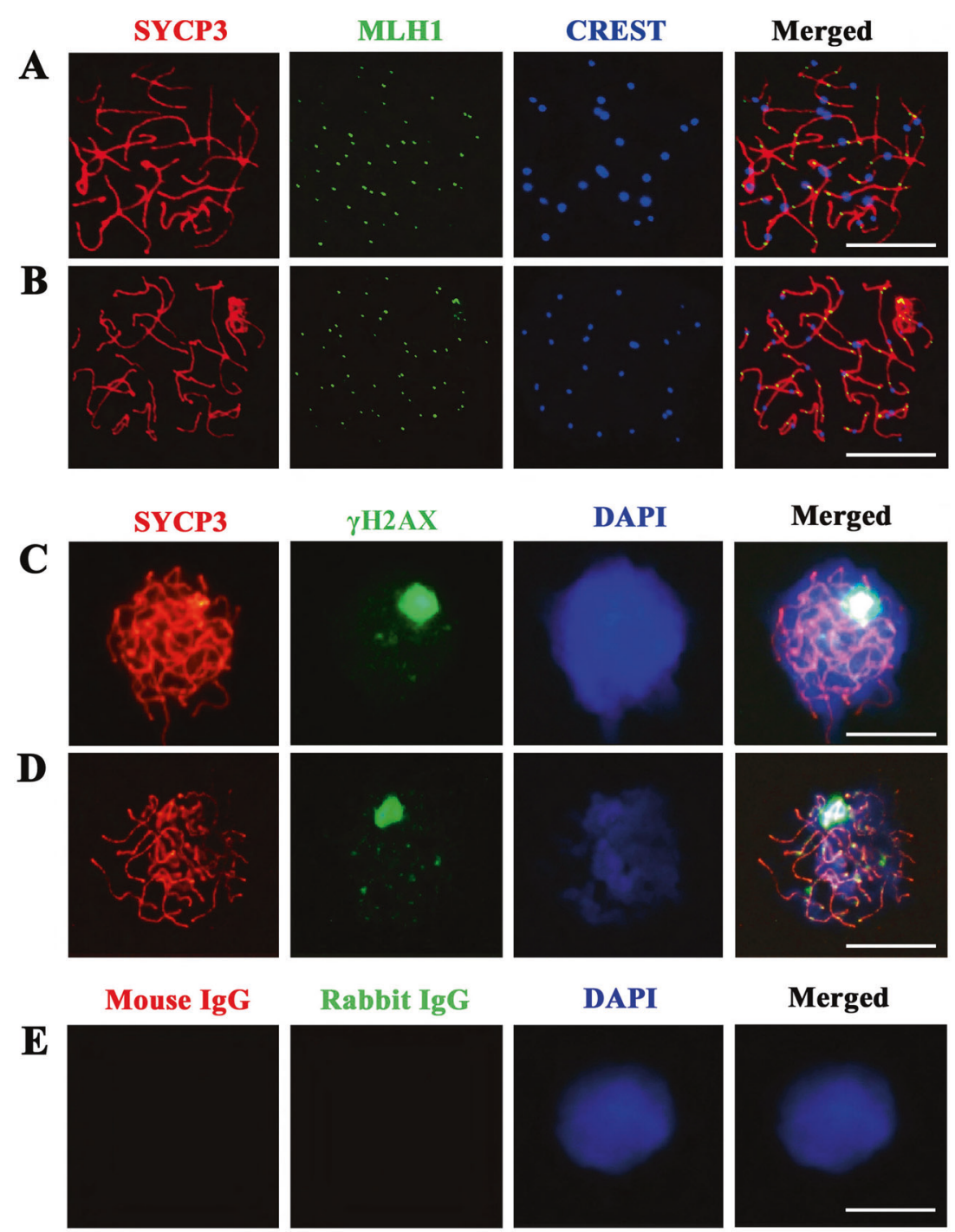

DAPI

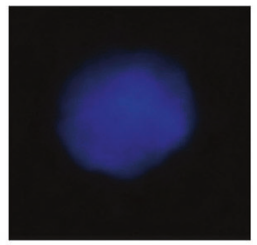

Merged

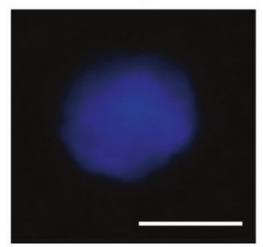

F

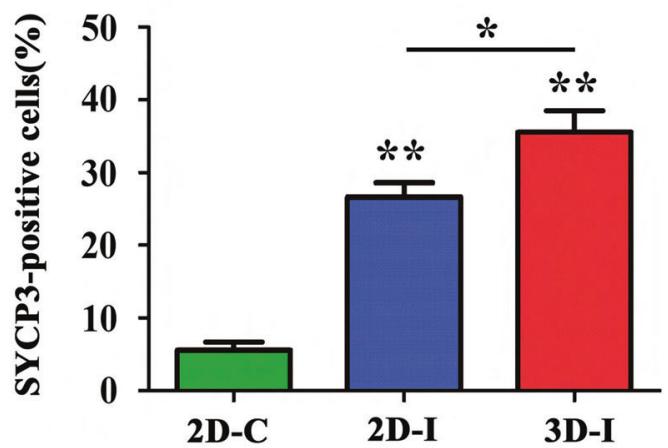

3D-negative (3D-N) group (human SSCs with Sertoli cells and matrigel but without defined medium), and 2D-control (2D-C) group (human SSCs without matrigel or defined medium) after induction for 15 days (Fig. 2c). These results reflect that 3D-I system has a better effect on inducing gene expression during the differentiation of human SSCs into spermatocytes and spermatids than the conventional 2D-I, 3D-C, 3D-N, or 2D-C system. In addition, SYCP1, SYCP3, TNP1, TNP2, PRM1, and PRM2 transcription was significantly enhanced in the cells of 3D-I system from day 5 to day 20 of induction (Fig. 2d), indicating that 3D-I system coaxes the differentiation of human SSCs into 
spermatocytes and spermatids at transcriptional levels in a time-dependent manner.

To observe the progression of meiosis including chromosomal synapsis and recombination during 3D-I system of human SSCs, meiotic chromatin spread and immunocytochemistry were conducted by examining the expression of SYCP3, CREST, MLH1, PIWIL1, PIWIL2, and $\gamma \mathrm{H} 2 \mathrm{AX}$ (phosphorylated H2A histone family member X), specific markers for meiotic cells [23, 24]. Meiotic chromatin spread analyses by triple immunostaining were performed with antibodies against SYCP3 for detecting axial/lateral elements of the synaptonemal complex, MLH1 for measuring the meiotic recombination frequency, and CREST for determining centromeric pairing of homologs [25].
Fig. 4 DNA contents and the expression of haploid markers in human SSCs-derived cells by 3D-I system. a FACS analysis of DNA contents in the cells generated from human SSCs in the 3D-I system for 5-20 days. Haploid (1C), diploid (2C), and tetraploid (4C) cells were shown. b, c FACS analysis of DNA contents in the cells generated from human SSCs in the 2D-I system at 20 days (b) and male germ cells from OA patients (c). The results shown in $\mathbf{a}-\mathbf{c}$ were presented from at least three independent experiments. d

Immunocytochemistry revealed the expression of ACR in human SSCs-derived haploid spermatids to show their purity sorted by FACS. Scale bar $=20$ $\mu \mathrm{m}$. e-h Immunocytochemistry revealed the expression of ACR (e) and PRM2 (g) in human SSCs-derived haploid cells sorted by FACS. Replacement of primary antibodies with isotype $\mathrm{IgGs}$ were utilized as negative controls $(\mathbf{f}, \mathbf{g})$. Scale bars in $\mathbf{e}-\mathbf{h}=10 \mu \mathrm{m}$. The results shown in $\mathbf{d}-\mathbf{h}$ were presented from at least three independent experiments
$\mathbf{A}$
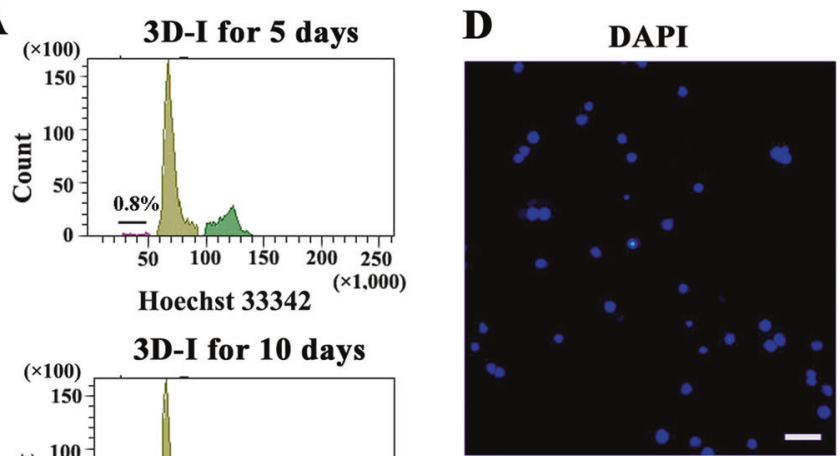

D
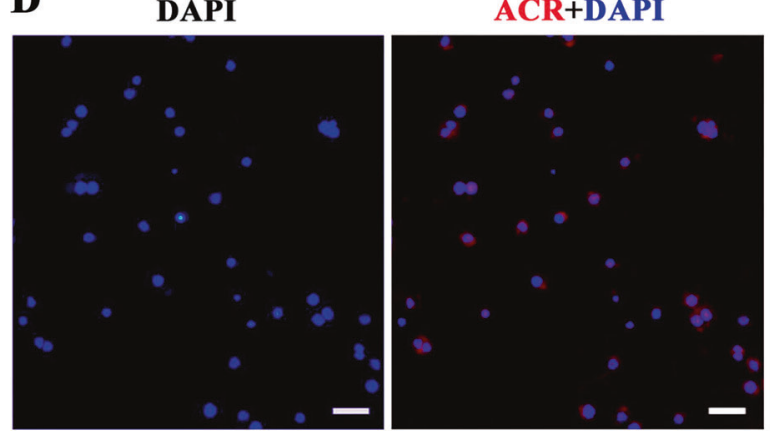

$\mathbf{E}$

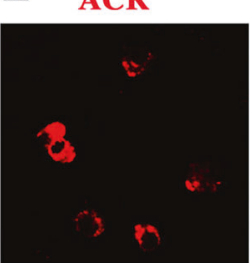

DAPI

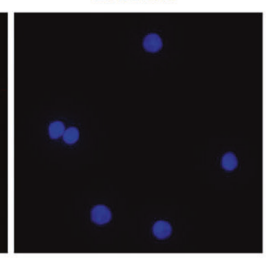

Merged

$200 \quad 250$
$(\times 1,000)$
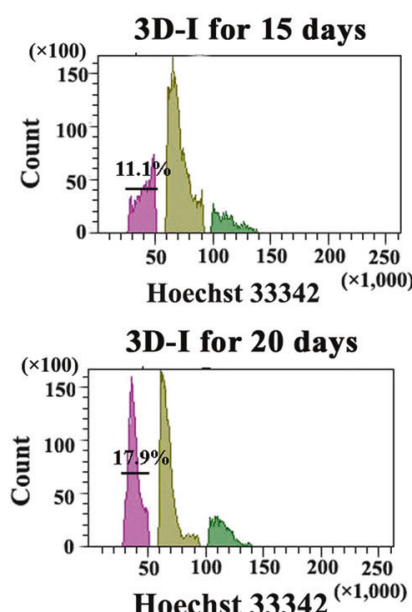

B

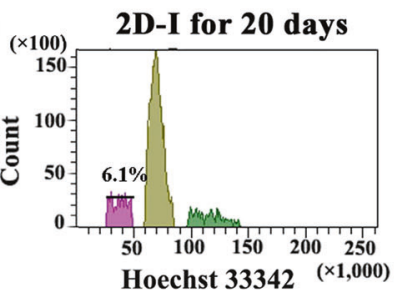

C

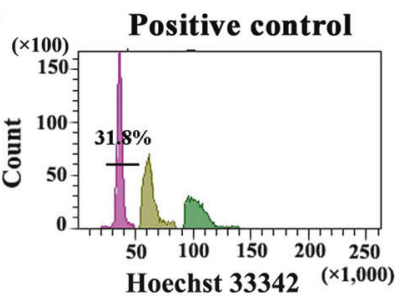

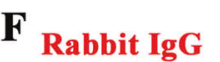
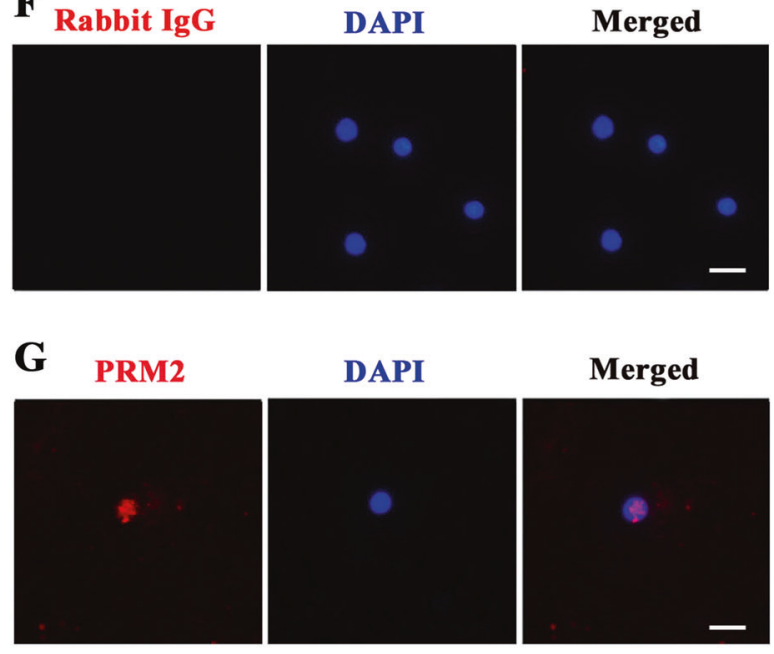

DAPI
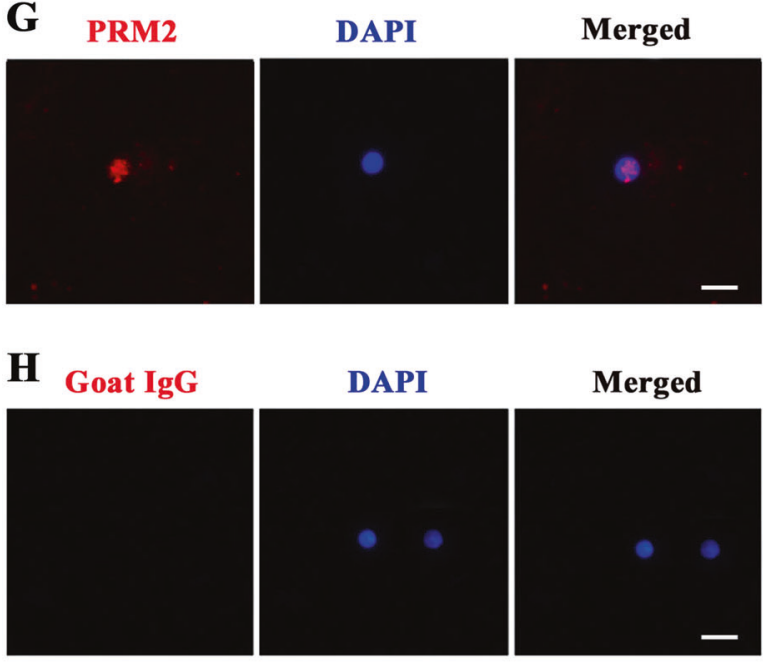

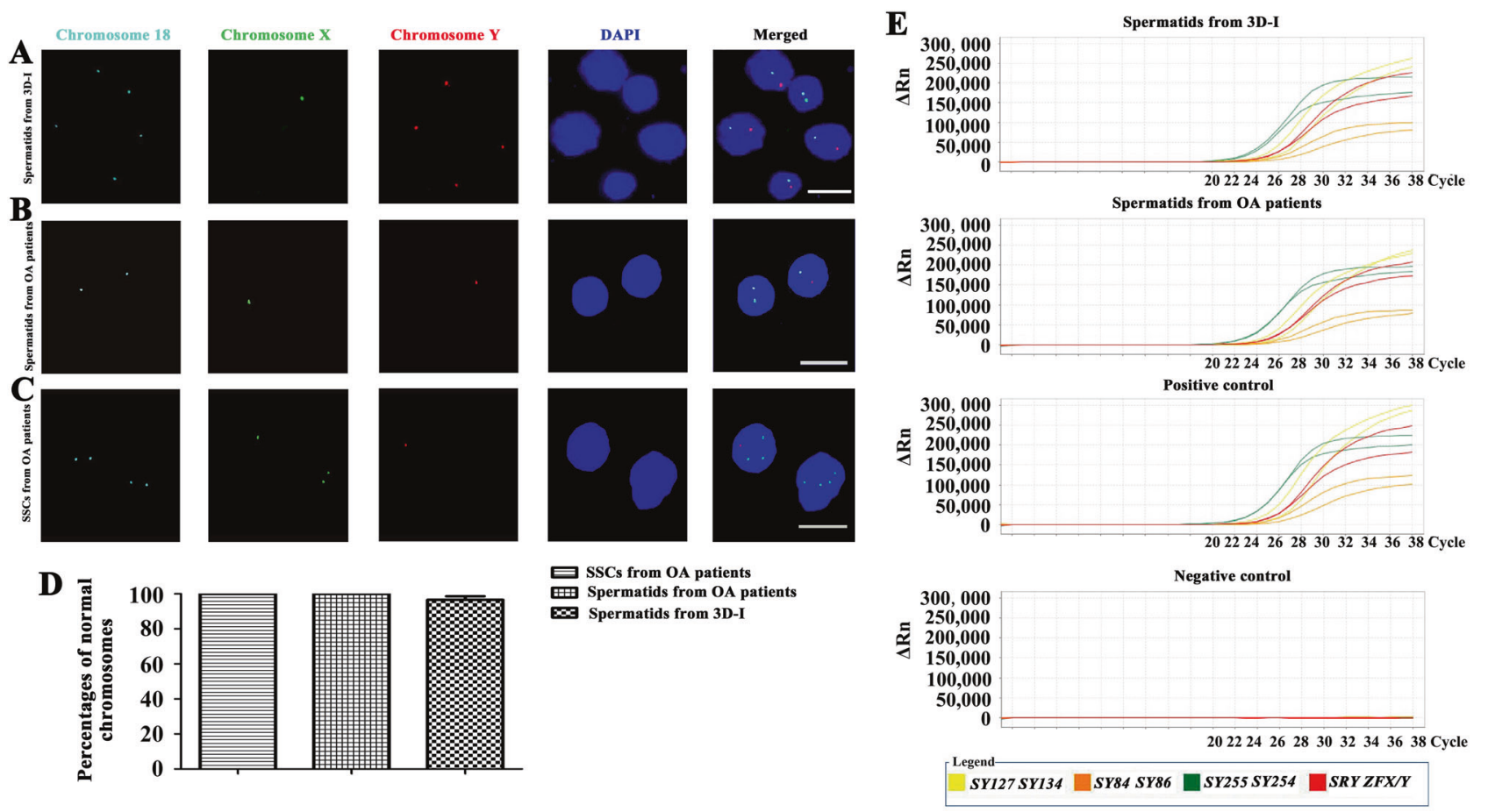

Fig. 5 Chromosomes and Y chromosome microdeletion analyses of human SSCs-derived spermatids.a-c Fluorescence in situ hybridization (FISH) revealed chromosomes $\mathrm{X}, \mathrm{Y}$, and 18 in round spermatids generated from human SSCs by the 3D-I system (a), round spermatids from OA patients (b), and human SSCs of OA patients (c). Scale bars in $\mathbf{a}-\mathbf{c}=10 \mu \mathrm{m}$. d FISH showed the percentages of normal chromosomes in round spermatids from human SSCs by 3D-I system, round spermatids from OA patients, and human SSCs from OA patients. The data shown in a-d were presented from at least three

Chromosomal spreads revealed that the cells were coexpressing SYCP3, CREST, and MLH1 in 3D-I group after induction for 20 days (Fig. 3a). As a positive control, the expression of SYCP3, CREST, and MLH1 was observed in pachytene spermatocytes of OA patients (Fig. 3b). Notably, 3D-I group induced a significantly higher percentage of SYCP3-positive cells compared to 2D-I group and $2 \mathrm{D}-\mathrm{C}$ groups (Fig. 3f). It has been reported that the distribution of $\gamma \mathrm{H} 2 \mathrm{AX}$ recapitulates meiotic progression in vivo [20]. The co-expression of $\gamma \mathrm{H} 2 \mathrm{AX}$ and SYCP3 in leptotene spermatocytes (Supplementary Fig. S4A, the 1st panel), zygotene spermatocytes (Supplementary Fig. S4A, the 2nd panel), and pachytene spermatocytes (Fig. 3c; Supplementary Fig. S4A, the 3rd panel) were detected in the cells of 3D-I system after 20 days of culture. The expression of $\gamma \mathrm{H} 2 \mathrm{AX}$ and SYCP3 in pachytene spermatocytes of OA patients served as a positive control (Fig. 3d), and replacement of primary antibodies with isotype $\operatorname{IgG}$ was used as a negative control showing no immunostaining in these cells (Fig. 3e). Considered together, the results reflect an association with DNA double-

independent experiments. e Multiplex real-time PCR displayed the expression of all eight specific STS markers from AZFa, AZFb and AZFc regions, including ZFX/ZFY, SRY, SY254, SY127, SY86, SY134, $S Y 84$, and SY255, in the round spermatids from human SSCs by 3D-I group (1st panel) and round spermatids of OA patients (2nd panel). DNA from normal human blood served as a positive control (3rd panel), and water substituting for DNA was used as a negative control (4th panel). The results shown in e were presented from three independent experiments

strand breaks (DSBs) in these cells. The disappearance of $\gamma \mathrm{H} 2 \mathrm{AX}$ from the autosome region was observed in the cells generated from human SSCs by 3D-I system at day 20 (Supplementary Fig. S4A, the 3rd panel), indicating that DSBs are repaired in these cells. In addition, we observed $\gamma \mathrm{H} 2 \mathrm{AX}$ accumulation on one chromosome pair, which is implicated to be the unsynapsed sex chromosomes [26]. Double immunostaining further revealed that PIWIL1 and PIWIL2 (Supplementary Fig. S4B), PIWIL1 and $\gamma \mathrm{H} 2 \mathrm{AX}$ (Supplementary Fig. S4C), PIWIL2 and SYCP3 (Supplementary Fig. S4D), were co-expressed in the cells derived from human SSCs by 3D-I system, suggesting that human SSCs could be induced to differentiate to spermatocytes of different stages. Replacement of primary antibodies with isotype IgGs was utilized as negative controls, and no immunostaining was seen in these cells (Supplementary Fig. S4E), thus verifying specific expression of these proteins mentioned above in spermatocytes. Altogether, these data indicate that our 3D-I system promotes the differentiation of human SSCs into various meiotic stages of spermatogenesis. 
A Hierarchical clustering of global gene expression

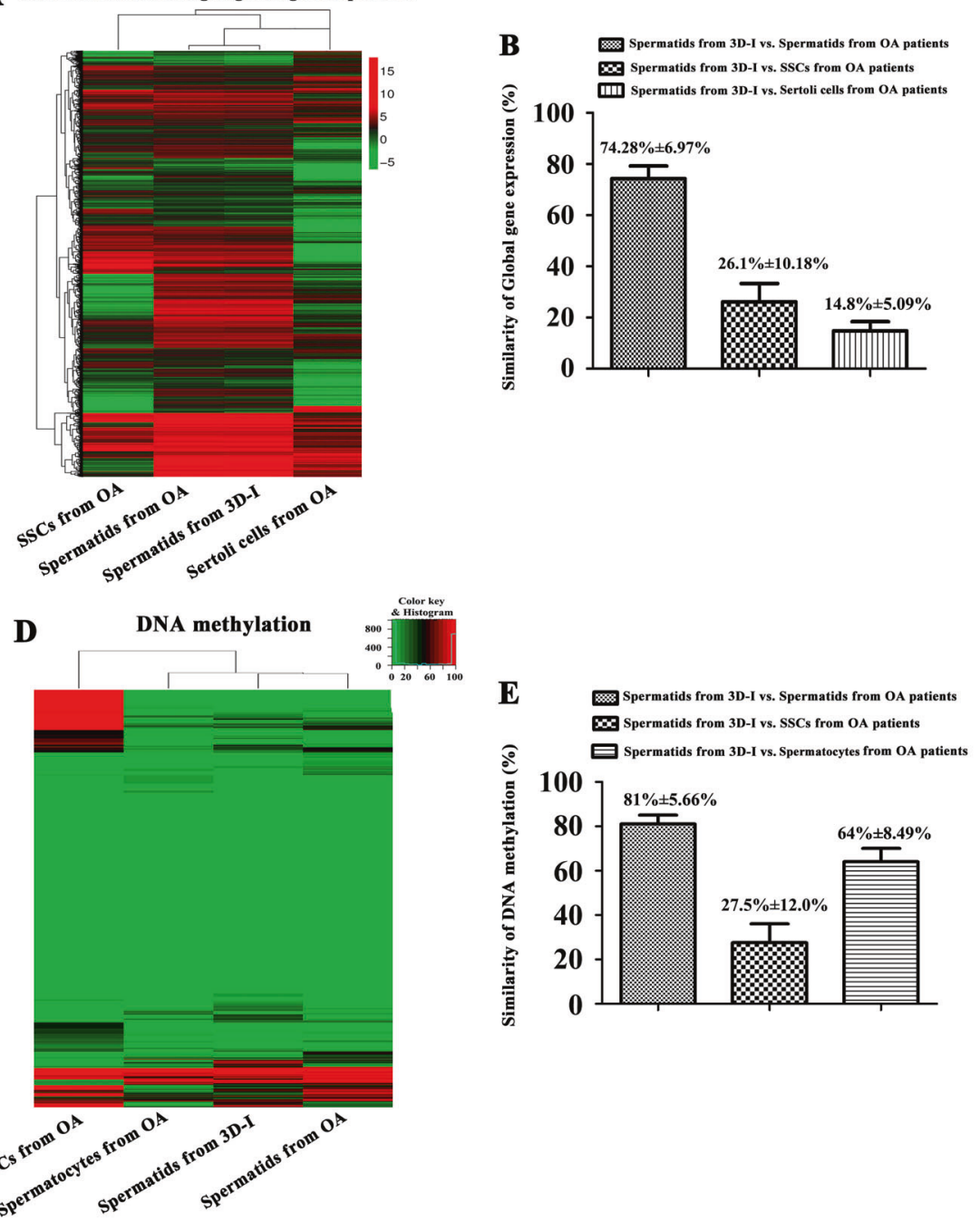

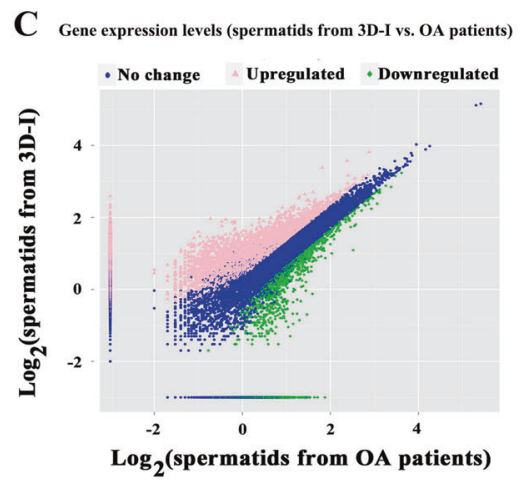

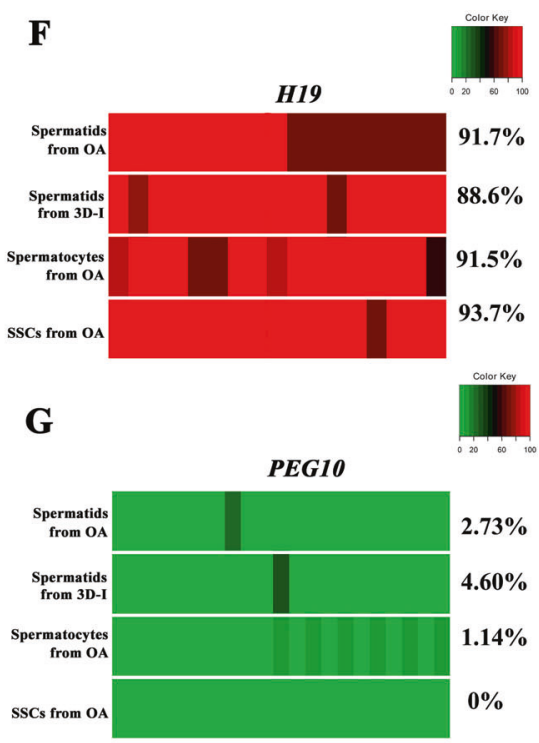

Fig. 6 Global gene expression and DNA methylation analyses of human SSCs-derived spermatids. a Hierarchical clustering analysis showed the global gene expression profiles of round spermatids derived from human SSCs by 3D-I system, round spermatids of OA patients, and human SSCs and human Sertoli cells of OA patients. b Single-cell RNA sequencing analysis revealed the similarities of large scale of gene expression among round spermatids derived from human SSCs by 3D-I system, round spermatids of OA patients, and human SSCs and human Sertoli cells of OA patients. c Scatter plot revealed global gene levels in human SSCs-derived spermatids and round spermatids from OA patients. d Hierarchical clustering analysis of bisulfite sequencing showed the similarities in the DNA methylation of

\section{Flow cytometry analysis of dynamic changes of DNA content in the cells derived from human SSCs to show meiosis and progressive post-meiotic spermatid development}

We further detected dynamic changes of DNA content (C) in the cells derived from human SSCs in our 3D-I system. Human spermatids are haploid (1C) after two rounds of meiotic division; spermatogonia and secondary round spermatids derived from human SSCs by 3D-I system, round spermatids of OA patients, pachytene spermatocytes, and human SSCs of OA patients. e Bisulfite sequencing analysis revealed the similarities of DNA methylation among round spermatids derived from human SSCs by 3D-I system, round spermatids of OA patients, and human SSCs and pachytene spermatocytes of OA patients. f, g Bisulfite sequencing illustrated the DNA methylation of imprinted genes, namely $H 19$ and PEG10, among round spermatids derived from human SSCs by 3D-I system, round spermatids of OA patients, pachytene spermatocytes of OA patients, and human SSCs of OA patients. The results shown in $\mathbf{a}-\mathbf{g}$ were presented from at least two independent experiments

spermatocytes are diploid (2C), whereas primary spermatocytes are tetraploid (4C). After hoechst 33342 staining, fluorescence-activated cell sorting (FACS) analysis was performed in human SSCs-derived cells by 3D-I system for 5-20 days (Fig. 4a) and 2D-I system for 20 days (Fig. 4b), and human freshly isolated testicular germ cells were utilized as a positive control (Fig. 4c). As shown in Fig. 4a, haploid cells were detected in the human SSCs-derived cells after 3D-I induction for 5 days, and notably, the percentages 
of haploid cells were increased significantly from 1.6 to $17.9 \%(17.9 \% \pm 0.06, n=7)$ (Fig. 4a; Supplementary Table S3) by 3D-I system from day 10 to day 20, which was obviously higher than the percentages of haploid cells derived from human SSCs by 2D-I system (Fig. 4b). In addition, we observed that the percentage of haploid cells at day 20 in the 3D-I system was higher than other time points, and thus the time point of 20 days was chosen to compare the differentiation efficiency of human SSCs. Immunocytochemistry displayed that more than $99 \%$ of these cells sorted by FACS using Hoechst 33342 were positive for Acrosin (ACR) (Fig. 4d). These data further suggest that 3D-I system promotes the differentiation of human SSCs into haploid spermatids.

ACR and Protamine 2 (PRM2) are generally regarded as markers for haploid cells. The developmental stages of FACS-separated haploid cells derived from human SSCs via 3D-I system were shown by the expression of ACRpositive cells (Fig. 4e) and PRM2-positive cells (Fig. 4g). Replacement of antibodies against ACR and PRM2 with isotype IgGs was used as negative controls, and no immunostaining was seen in the cells derived from human SSCs by 3D-I system (Fig. 4f, h), thus verifying specific expression of ACR and PRM in these cells. Considered together, these results implicate that 3D-I system stimulates human SSC differentiation into haploid spermatids.

\section{Round spermatids derived from human SSCs by 3D-I system had normal chromosomes and assumed similar transcriptome and DNA methylation with normal round spermatids}

To detect chromosome numbers in round spermatids derived from human SSCs by our 3D-I system, fluorescence in situ hybridization (FISH) analyses were conducted for several chromosomes, including $\mathrm{X}, \mathrm{Y}$, and 18. Notably, $97 \%$ of round spermatids exhibited the normal constitution of the sex chromosomes and autosome (Fig. 5a, d), except $3 \%$ of these cells had both $\mathrm{X}$ and $\mathrm{Y}$ chromosomes (Figure S5A). As positive controls, $100 \%$ of round spermatids (Fig. 5b, d) and human SSCs (Fig. 5c, d) from OA patients displayed normal sex chromosomes and autosome, whereas replacement of chromosome probes with PBS served as negative controls and no staining was observed (Supplementary Fig. S5B). Taken together, these data reflect that round spermatids generated from human SSCs by 3D-I system have normal chromosome numbers.

Multiplex real-time PCR was used to assess whether there were $\mathrm{Y}$ chromosome microdeletions in round spermatids derived from human SSCs by the 3D-I system. Eight specific STS markers from AZFa, AZFb and AZFc regions, including ZFX/ZFY, SRY, SY254, SY127, SY86, SY134, $S Y 84$, and $S Y 255$, were all detected in round spermatids derived from human SSCs by our 3D-I system (Fig. 5e, the 1 st panel) and round spermatids obtained from OA patients (Fig. 5e, the 2nd panel). DNA from normal human blood served as a positive control (Fig. 5e, the 3rd panel), and water substituted for DNA as a negative control (Fig. 5e, the 4 th panel). The results implicate that the round spermatids derived from human SSCs exclude Y chromosome microdeletions.

RNA sequencing was carried out to compare the global gene profiles of round spermatids derived from human SSCs by 3D-I system, normal round spermatids from OA patients, and human SSCs and Sertoli cells from OA patients. There were $22,128,794$ reads, and $67.73 \%$ of mapped reads in round spermatids derived from human SSCs. In parallel, 24,116,444 reads and $70.70 \%$ of mapped reads were detected in round spermatids of OA patients, and $22,724,550$ reads and $79.42 \%$ of mapped reads were found in human SSCs derived from OA patients. In addition, $22,730,054$ reads and $76.01 \%$ of mapped reads were detected in human Sertoli cells derived from OA patients. In total, 13,083 genes were found in round spermatids derived from human SSCs by 3D-I system (Supplementary Fig. S6A), whereas 11,732 genes were identified in round spermatids of OA patient and 18,469 genes were detected in human SSCs (Supplementary Fig. S6A). There were 18,331 genes found in human Sertoli cells (Supplementary Fig. S6A). Hierarchical clustering analyses revealed the global gene expression profiles among round spermatids derived from human SSCs by 3D-I system, normal round spermatids of OA patients, and human SSCs and human Sertoli cells obtained from OA patients (Fig. 6a). It was worth noting that there was a similarity of $74.28 \% \pm 6.97 \%$ of genes between round spermatids generated from human SSCs by 3D-I system and normal round spermatids of OA patients (Fig. 6b, c). In contrast, there was only $14.8 \% \pm$ $5.09 \%$ of similarity in the global genes between round spermatids derived from human SSCs by 3D-I system and human Sertoli cells of OA patients (Fig. 6b), and 26.1\% \pm $10.18 \%$ of similarity existed in the large scale of genes between round spermatids derived from human SSCs by 3D-I system and human SSCs of OA patients (Fig. 6b, c; Supplementary Fig. S6B). These results implicate that there is a similarity in the majority of global transcriptome profiles of round spermatids derived from human SSCs by 3D-I system and normal round spermatids of OA patients.

We further utilized real-time PCR to verify the validity of RNA sequencing data. A number of hallmarks for haploid spermatids were selected in terms of their fold changes in both types of round spermatids. Real-time PCR showed that the transcripts of $A C R, A C R B P, H N R N P, D K K L 1$, IZUMO1, TNP2, SPA17, and PGK2 were differentially expressed between round spermatids derived from human SSCs by 3D-I system and normal round spermatids 
Fig. 7 Fertilization and development capacities of human SSCs-derived spermatids and H3K9me3 expression of embryos from round spermatids generated from human SSCs. a-c Phasecontrast microscope showed the morphology of embryos with two pronuclei (a), embryos developing into the two-cell (b), and four and eight-cell (c) stages. Scale bars in $\mathbf{a}-\mathbf{c}=20$ $\mu \mathrm{m}$. The results shown in $\mathbf{a}-\mathbf{c}$ were presented from at least five independent experiments. d Hierarchical clustering analysis of single-cell RNA sequencing showed the similarities in the global gene expression profiles between the embryos derived from mouse oocytes fertilized with human round spermatids of human SSCs by 3D-I and the embryos derived from mouse oocytes fertilized with mouse round spermatids. e Scatter plot revealed large scale of gene expression levels in the embryos derived from human round spermatid and mouse round spermatid. The data shown in $\mathbf{d}$ and $\mathbf{e}$ were presented from at least two independent experiments. f-h Double immunocytochemistry revealed expression of $\mathrm{H} 3 \mathrm{~K} 9 \mathrm{me} 3$ and human nuclear antigen (HumNuc) in the embryos derived from human round spermatid (f) and human SSCs (g). h Replacement of primary antibodies with isotype IgGs in the embryos derived from human round spermatid served as negative controls. Scale bars $\mathbf{f}-\mathbf{h}=25 \mu \mathrm{m}$. The results shown in $\mathbf{f}-\mathbf{h}$ were presented from at least three independent experiments
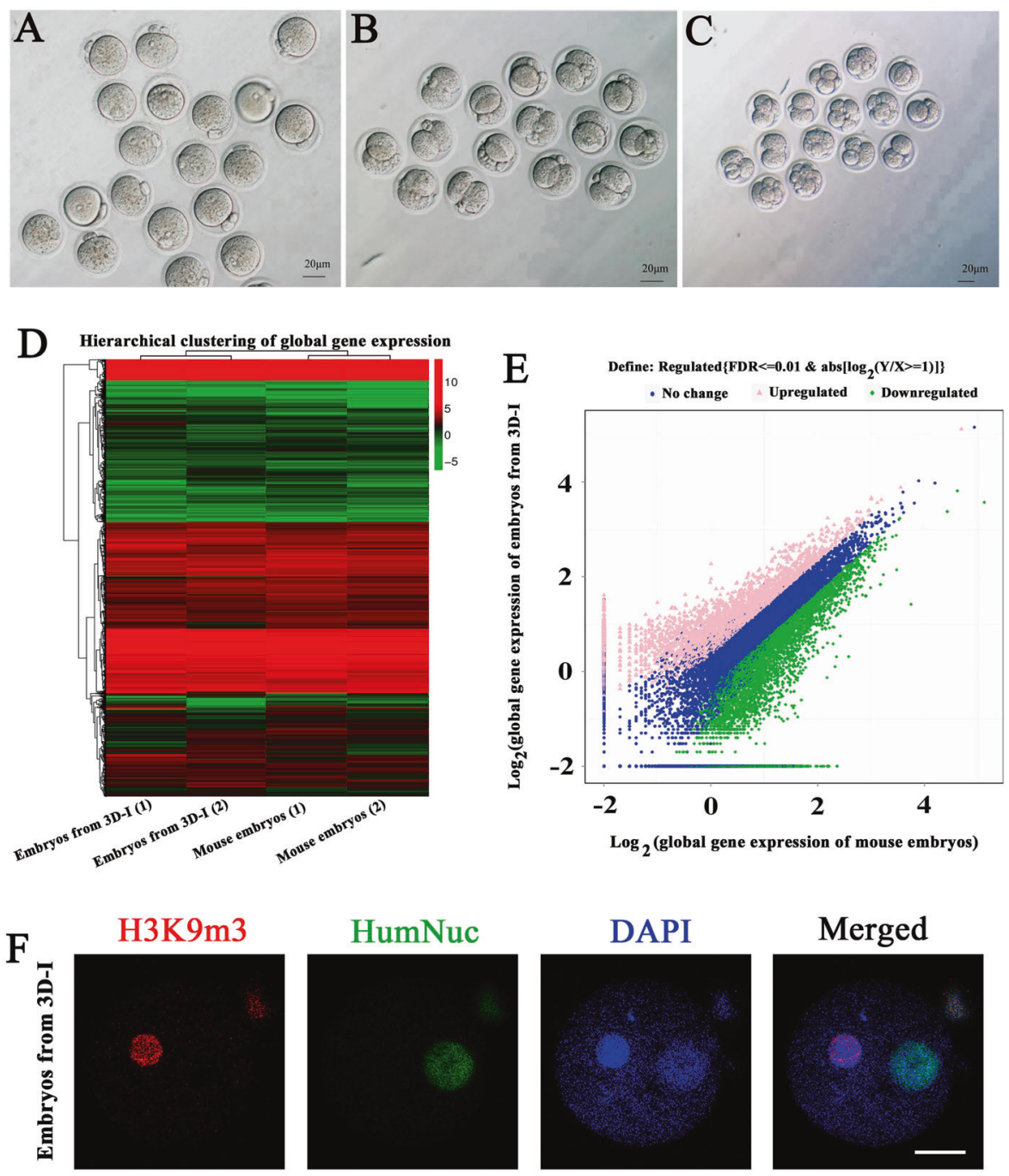

HumNuc
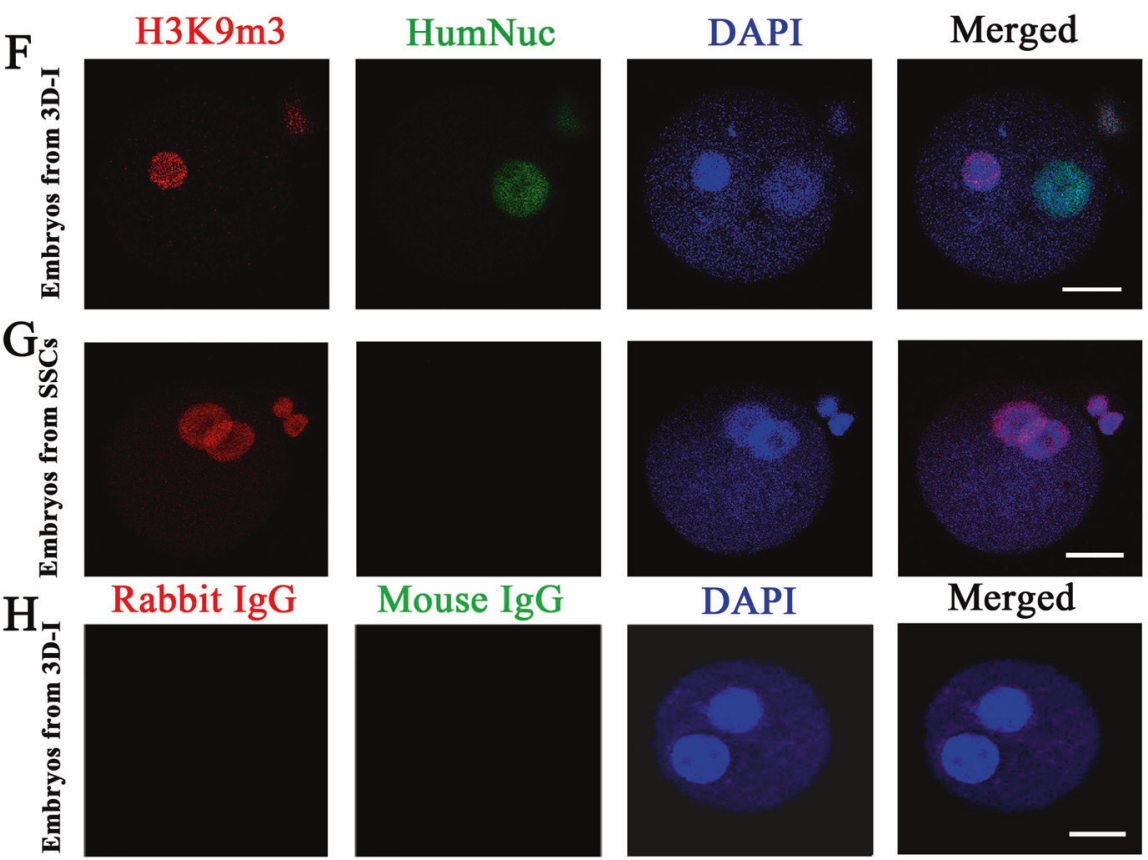

(Supplementary Fig. S6D), whereas there was no significant difference in the expression of TNP1, PRM1, PRM2, $S P E F 2$, and SPATA19 between these two types of cells (Supplementary Fig. S6D). The differential expression of ACR, ACRBP, HNRNP, DKKL1, IZUMO1, TNP2, SPA17, and $P G K 2$ genes in round spermatids derived from human SSCs by 3D-I system and normal round spermatids might be attributed to the effects of factors and hormone used in the 3D-I system. The expression patterns of all of these genes determined by real-time PCR were fully consistent with the data of RNA sequencing.

Bland-Altman plot can be utilized to verify the consistency of two experimental methods [27, 28]. As shown in Supplementary Fig. S6C, all of the genes, including TNP1, 
TNP2, PRM1, PRM2, SPATA19, SPA17, PGK2, IZUMO1, HNRNP, SPEF2, ACR, ACRBP, DKKL1, assumed 95\% confidence intervals, which reflected a very high consistency in gene expression profiles obtained from RNA sequencing and real-time PCR. Taken together, these results confirmed the quality and authenticity of our RNA sequencing data, suggesting that round spermatids derived from human SSCs in vitro have a similarity in the global transcriptome compared to normal round spermatids in vivo.

To compare the global DNA methylation profiles of round spermatids derived from human SSCs by 3D-I system, normal round spermatids from OA patients, and human SSCs and pachytene spermatocytes from OA patients, bisulfite sequencing was carried out. There were 15,529,793 total read pair and $62.30 \%$ of mapped reads in round spermatids derived from human SSCs. In parallel, $25,611,460$ total read pair and $70.30 \%$ of mapped reads were detected in round spermatids of OA patients, whereas $25,385,252$ total read pair and $71.30 \%$ of mapped reads were identified in human SSCs from OA patients. In addition, $21,165,486$ total read pair and $66.80 \%$ of mapped reads were found in human pachytene spermatocytes from OA patients. Hierarchical clustering analyses further revealed various similarity in DNA methylation profiles among round spermatids derived from human SSCs by 3D-I system, normal round spermatids from OA patients, and human SSCs and pachytene spermatocytes from OA patients (Fig. 6d). Interestingly, there was $81 \% \pm 5.66 \%$ and $64 \% \pm 8.49 \%$ of similarity in DNA methylation between round spermatids derived from human SSCs by 3D-I system and normal round spermatids and pachytene spermatocytes of OA patients (Fig. 6e), respectively. Conversely, only $27.50 \% \pm 12.00 \%$ of similarity existed in DNA methylation between round spermatids derived from human SSCs by 3D-I system and human SSCs of OA patients (Fig. 6e). Taken together, these results suggest that round spermatids derived from human SSCs in vitro have a similarity in the majority of global DNA methylation compared to normal round spermatids in vivo. The methylation levels of male and female imprinted genes, including H19 and PEG10, respectively, were further analyzed among round spermatids derived from human SSCs by 3D-I system, normal round spermatids from OA patients, and human SSCs and pachytene spermatocytes from OA patients. As shown in Fig. 6f and g, DNA methylation of H19 and PEG10 genes in round spermatids derived from human SSCs by 3D-I system was $88.6 \%$ and $4.6 \%$, which was comparable to the DNA methylation of $H 19$ and PEG10 genes in normal round spermatids of OA patients. DNA methylation of $H 19$ gene in round spermatids derived from human SSCs by 3DI system was significantly lower than DNA methylation of H19 gene in human SSCs from OA patients (Fig. 6f), whereas DNA methylation of PEG10 gene in round spermatids derived from human SSCs by 3D-I system was significantly higher than human SSCs from OA patients (Fig. 6g).

\section{Round spermatids generated from human SSCs by 3D-I system could fertilize mouse oocytes to form embryos with developmental capacity}

Round spermatid microinjection (ROSI) was performed to determine the fertilization potential of human round spermatids generated from human SSCs using 3D-I system, round spermatids and human SSCs isolated from OA patients. The procedure of ROSI was shown in Supplementary Fig. S7A-F. In the microinjection of human round spermatids derived from human SSCs by 3D-I system, $50 \%$ of these spermatids could fertilize mouse oocytes to form pronuclear embryos (Fig. 7a; Supplementary Table S4) and developed into the 2-cell stage (Fig. 7b; Supplementary Table S4), and notably, $17.86 \%$ of the embryos were able to develop into 4-cell and 8-cell stages (Fig. 7c; Supplementary Table S4). In the microinjection of human round spermatids of OA patients, $50 \%$ of them fertilized mouse oocytes and survived to develop into the 2-cell stage (Supplementary Table S4), and $12.5 \%$ of the embryos developed into 4-cell and 8-cell stages (Supplementary Table S4). As a positive control, $100 \%$ of mouse round spermatids could fertilize mouse oocytes and developed into the 2-cell stage (Supplementary Table S4), and $42.1 \%$ of the embryos developed into 4-cell and 8-cell stages (Supplementary Table S4). As a negative control, none of the embryos derived from human SSCs from OA patients and mouse oocytes developed into 2-cell, 4-cell, or 8-cell stages (Supplementary Table S4).

Moreover, global gene expression profiles were compared between the embryos generated from the microinjection of round spermatids derived from human SSCs by 3D-I system into mouse oocytes and mouse embryos obtained from microinjection of mouse spermatids into mouse oocytes. RNA sequencing revealed that there were $22,866,771$ total reads and $55.08 \%$ of mapped reads in embryos derived from mouse oocytes fertilized with round spermatids of human SSCs by 3D-I system; in parallel, $22,881,394$ total reads and $63.60 \%$ of mapped reads were detected in the embryos derived from mouse oocytes fertilized with mouse round spermatids. In total, 14,445 genes were found in embryos derived from mouse oocytes fertilized with human round spermatids of human SSCs by 3D-I system, whereas 14,943 genes were identified in the embryos derived from mouse oocytes fertilized with mouse round spermatids. Hierarchical clustering analyses revealed the differences in global transcriptome profiles of embryos derived from mouse oocytes fertilized with human round spermatids of human SSCs by 3D-I system and embryos 
derived from mouse oocytes fertilized with mouse round spermatids (Fig. 7d). It was worth noting that $41.7 \%$ of global genes had significant difference in the expression between those two embryo groups (Fig. 7e), indicating that more than $41 \%$ of human paternal genome is transcriptionally activated in the hybrid embryos derived from human round spermatids of human SSCs by 3D-I system and mouse oocytes.

To verify the fertilization of human round spermatids generated from human SSCs by 3D-I system with mouse oocytes and exclude parthenogenetic activation of mouse oocytes themselves, double immunocytochemistry was carried out using antibodies against human nuclear antigen (HumNuc) and $\mathrm{H} 3 \mathrm{k} 9$ trimethylation (H3K9me3). The embryos derived from mouse oocytes fertilized with human round spermatids of human SSCs by 3D-I system were positive for both human anti-HumNuc and anti-H3K9me3 (Fig. 7f), reflecting human gene activation in the 3D-I system-derived embryos. In contrast, the embryos derived from mouse oocytes fertilized with human SSCs were positive for H3K9me3 protein but were negative for HumNuc protein (Fig. $7 \mathrm{~g}$ ). Replacement of primary antibodies with isotype IgGs was used as negative controls, and no immunostaining was seen in embryos derived from round spermatids of human SSCs by 3D-I system (Fig. 7h), thus excluding the parthenogenetic activation of mouse oocytes in the embryos generated from fertilization with human round spermatids generated from human SSCs by 3D-I system.

To further determine the dynamic changes during the development of embryos generated from human SSCsderived round spermatids, H3K9me3 was applied to label the maternal genomes. As shown in Supplementary Fig. S7G and $\mathrm{H}$, maternal pronuclei were positive for $\mathrm{H} 3 \mathrm{~K} 9$ me3 whereas its staining in male pronucleus was hardly detectable. Sperm chromatin began to de-condense immediately after fertilization, and male and female pronuclei were stained for $\mathrm{H} 3 \mathrm{~K} 9 \mathrm{me} 3$ at $6 \mathrm{~h}$ after fertilization (Supplementary Fig. S7G). At 2-cell stage, both nuclei of embryos stained positively for H3K9me3 (Supplementary Fig. S7H). Replacement of primary antibody with isotype IgG was used as a negative control, and no immunostaining was seen in embryos derived from human SSCs by 3D-I system (Supplementary Fig. S7I), thus verifying specific expression of $\mathrm{H} 3 \mathrm{~K} 9 \mathrm{me} 3$ protein in these cells. Together, these results clearly implicated that human round spermatids derived from human SSCs by 3D-I system have both fertilization and development capacities.

\section{Discussion}

Functional haploid spermatids have recently been generated in vitro from mouse ES cells [20]. However, complete spermatogenesis in vitro to obtain functional male gametes has not yet been achieved in humans. It has been shown that human primordial germ cell-like cells (hPGCLCs) can be generated from human iPS cells in vitro [29], and SOX17 has been shown to be a key regulator for hPGCLC specification from human pluripotent stem cells, whereas BMIMPI suppresses endodermal genes in the process of hPGCLC specification [30]. Integrin- $\beta 3$ and SSEA1 have been identified as markers to separate PGCLCs from mouse ES cells and iPS cells [31]. In addition, overexpression of transcription factors, namely Blimp1, Prdm14, and Tfap2c, induces the generation of PGCLCs which can achieve spermatogenesis in vitro [32]. Nevertheless, it remains to be determined whether hPGCLCs are able to be differentiated into male gametes in vitro. It has recently been demonstrated that functional spermatids [20] and mature oocytes [33] could be successfully generated in vitro from mouse pluripotent stem cells. However, generating functional spermatids has not yet been achieved in humans in vitro. Here we have for the first time demonstrated successful and efficient generation of human functional spermatids, conforming to the gold standards of the in vitro-derived germ cells, from human SSCs by 3D-I system in vitro. Specifically, we have revealed that human SSCs entered meiosis in vitro, underwent several key processes of the in vivo meiosis (e.g., chromosomal synapsis and recombination, and DSB repair), and finally differentiated into haploid spermatids with normal chromosome numbers. The spermatids derived from the 3D-I system assumed similarities in global gene profiles and DNA methylation when compared to round spermatids in vivo and they could successfully fertilize mouse oocytes by ROSI to form embryos with developmental potentials. This study thus illustrates the recapitulation of spermatogenesis in culture and proves the functionality of spermatids generated from human SSCs in vitro.

Spermatogenesis begins with SSC. The identity of the initial cells is critical to evaluate whether the whole process of human spermatogenesis can be achieved in vitro. Although it has been reported that spermatids can be obtained from non-obstructive azoospermic patients, the starting cells were testicular cell mixture without further cell isolation [9, 34]. The CD49-positive cells are separated from non-obstructive azoospermic patients; [8] however, CD49 is not a suitable marker of human SSCs because it is also expressed in human Sertoli cells [21]. We have reported the generation of haploid spermatids from germ cells of cryptochid patients, but the initial cells were not separated by MACS or FACS and they might be contaminated with spermatocytes or spermatids. We have demonstrated that GPR125 is a hallmark for human SSCs [21] and human SSC line generated from human GPR $125^{+}$ spermatogonia assume functionality of primary human SSCs, as they can colonize and proliferate in vivo in the 
recipient mice after xenotransplantation [35]. To complete the whole process of human spermatogenesis in vitro, we separated human GPR $125^{+}$spermatogonia from the testes of OA patients using MACS. Notably, human GPR $125^{+}$ spermatogonia possessed phenotypic characteristics of human primary SSCs, as evidenced by our observations that these cells were expressing a number of markers for SSCs, including GPR125, GFRA1, UCHL1, PLZF, and MAGEA4, but not spermatocyte or spermatid markers, e.g., SYCP1, SYCP3, TNP1, TNP2, PRM1, PRM2, and ACR. These results thus reflect that the starting cells we utilized for this study were indeed human SSCs with high purity without contamination of human spermatocytes or spermatids.

To establish an efficient approach for in vitro human spermatogenesis, we established a 3D-I system utilizing matrigel matrix to coax the differentiation of human SSCs into spermatids. This 3D system imitated the testicular microenvironment or niche, which is far superior to the conventional 2D method to support germ cell differentiation. Re-aggregation in a matrix is likely to re-establish germ cell and Sertoli cell contact that stimulates the development of germ cells. Moreover, embedding of cells in a gel provides a cubic structure to mimic the extracellular matrix of proteins and other biological molecules [36]. As basement membrane components seem to be physiologically appropriate for the 3D culture of testicular cells, matrigel matrix provides a 3D environment for germ cell differentiation. Previous studies have indicated that spermatogenic efficiency and testis tubule formation can be improved by the addition of matrigel [37-39]. Therefore, matrigel matrix was selected in this study. Sertoli cells are capable of self-assembling into seminiferous cord-like structures through a cell-intrinsic property, and matrigel can induce the formation of seminiferous tubules [40]. We found that 3D culture system with matrigel matrix and coculture of human Sertoli cells could significantly enhance the efficiency of human SSC differentiation into spermatids in vitro compared to the 2D culture systems without Sertoli cells. The transcript of SYCP3 was extremely higher in the 3D-I system than the 2D-I or 2D-C systems, whereas the level of SYCP3 protein was relatively higher in the 3D-I system compared to the $2 \mathrm{D}-\mathrm{I}$ or $2 \mathrm{D}-\mathrm{C}$ systems, which might be due to the fact that there was a delay with translation of SYCP3 in the 3D-I system.

To improve the efficiency of human SSC differentiation, we optimized the differentiation-medium. It has been reported that knockout serum replacement (KSR), retinoic acid (RA), stem cell factor (SCF), bone morphogenetic protein 4 (BMP4), and gonadotropins have an essential role in germ cell differentiation from mouse testicular cells and iPS cells. For instance, KSR has been shown to be effective for the in vitro production of spermatids in an organ culture of neonatal mouse testes [18], whereas RA can activate
Stra8 gene expression and SCF/KIT signaling pathway to initiate the meiosis [41] and induce mouse iPS cells to differentiate into male germ cells [42]. SCF has an important role in regulating SSC proliferation and differentiation and it promotes the generation of round spermatids from mouse spermatogonia [19]. We have previously shown that RA and SCF stimulate the differentiation of testis cells enriched in human spermatogonia into post-meiotic male germ cells [10]. BMP4 has an impact on both proliferation and differentiation of mouse SSCs [43-46]. Recently, it has been shown that SCF and testosterone are effective for inducing the differentiation of mouse ES cells into male germ cells [20]. On the basis of these findings in our pilot experiments, we selected the inclusion of KSR, RA, SCF, BMP4, and testosterone in our defined medium, which significantly increased the differentiation efficiency of human SSCs into round spermatids by over 10-fold increase, up to $17.9 \%$ compared to differentiation efficiency of $1.71 \%$ as previously reported by Sato et al. [16].

To further assess the safety and functionality of haploid spermatids derived from human SSCs by 3D-I system, we detected their chromosomes, $\mathrm{Y}$ chromosome microdeletions, transcriptome and DNA methylation, using FISH, multiplex real-time PCR, RNA sequencing, and bisulfite sequencing, respectively. Notably, human SSCs-derived round spermatids assumed normal chromosomes and similar global gene profiles and DNA methylation with normal round spermatids and excluded $\mathrm{Y}$ chromosome microdeletions. Of unusual significance, haploid spermatids derived from human SSC by 3D-I system had the potentials to fertilize mouse oocytes to form embryos that were capable of developing into 4-cell and 8-cell stages, whereas none of the embryos obtained from human SSCs and mouse oocytes developed into 4- and 8-cell stages. In summary, we have demonstrated that 3D-I system are efficient in promoting the differentiation of human SSCs from OA patients into haploid spermatids with phenotypic features, DNA content, normal chromosome numbers, global transcriptome, DNA methylation, and fertilization and development capacity. There are millions of azoospermia patients worldwide. This study offers an ideal approach to generate human functional haploid spermatids from SSCs for in vitro fertilization. Most significantly, human SSCs-derived spermatids could provide male gametes for treating male infertility, particularly in azoospermic men who are unable to generate sperm but have SSCs.

\section{Materials and methods}

\section{Procurement of testicular biopsies from obstructive azoospermia}

Testicular biopsies were obtained from 80 obstructive azoospermia (OA) patients of 13 to 47 years old from 
October 2014 to March 2017. All OA patients with normal spermatogenesis in vivo were caused by inflammation and vasoligation but not by congenital absence of the vas deferens or other diseases including cancer. This study was approved by The Institutional Ethical Review Committee of Ren Ji Hospital (license number of ethics statement: 201201), Shanghai Jiao Tong University School of Medicine. The testicular tissues were immediately placed aseptically in Dulbecco modified Eagle medium (DMEM) (Gibco) containing antibiotic with $1 \%$ penicillin and streptomycin (Gibco).

\section{Histological examination}

Testicular tissues from $10 \mathrm{OA}$ patients were fixed in $4 \%$ in paraffin (PFA), embedded in paraffin, and sectioned at $5 \mu \mathrm{m}$ thickness. The sections were stained with hematoxylin and eosin (H\&E), and they were examined under a microscope.

\section{Isolation of human SSCs from OA patients}

Testicular tissues from 60 OA patients for at least 7 experiments were washed three times in DMEM-containing antibiotics penicillin and streptomycin. Human GPR125positive spermatogonia were separated using procedures as previously described [21]. In brief, seminiferous tubules were isolated from human testis biopsies using $2 \mathrm{mg} / \mathrm{ml}$ collagenase IV (Sigma) and $1 \mu \mathrm{g} / \mu \mathrm{l}$ DNase I (Gibco). Human testicular cells were obtained using a second enzymatic digestion with $4 \mathrm{mg} / \mathrm{ml}$ collagenase IV, $2.5 \mathrm{mg} /$ $\mathrm{ml}$ hyaluronidase (Sigma), $2 \mathrm{mg} / \mathrm{ml}$ trypsin (Sigma), and 1 $\mu \mathrm{g} / \mu \mathrm{l}$ DNase I. For differential plating, cells were seeded into culture plates in DMEM/F-12 (Gibco) supplemented with $10 \%$ FBS (Hyclone) and incubated at $34{ }^{\circ} \mathrm{C}$ in $5 \% \mathrm{CO}_{2}$ for $12 \mathrm{~h}$. After incubation, Sertoli cells attached to the culture plates, whereas male germ cells were remained in suspension and collected by centrifugation at $1000 \mathrm{rpm}$ for $5 \mathrm{~min}$. GPR125-positive spermatogonia were separated by magnetic-activated cell sorting (MACS) using an antibody to GPR125 at a dilution of 1:40 pursuant to the procedures in accordance with MACS instruction (Miltenyi Biotec).

\section{Reconstitution of testicular cells in matrigel matrix}

Human Sertoli cells at the third passage of culture were treated with $10 \mathrm{ng} / \mathrm{ml}$ mitomycin C (MMC, Sigma-Aldrich) at $37{ }^{\circ} \mathrm{C}$ in a humidified incubator for $2 \mathrm{~h}$ to inhibit their division, and they were washed six times with DMEM/F12. The MMC-treated Sertoli cells were digested with $0.25 \%$ trypsin (Sigma) for $2 \mathrm{~min}$, and they were collected by centrifugation at $1514 \mathrm{rpm}$ for $5 \mathrm{~min}$ and suspended in DMEM/F-12.
In total, $10^{6}$ cells (freshly isolated GPR125-positive spermatogonia from 4-8 OA patients for each differentiation experiment and inactivated human Sertoli cells with a ratio of $1: 3$ in $0.4 \mathrm{ml}$ DMEM/F-12) were mixed with $0.4 \mathrm{ml}$ matrigel $(9 \mathrm{~m} / \mathrm{ml}$, Corning), and they were plated into 12 well culture dishes and incubated at $34{ }^{\circ} \mathrm{C}$ in a humidified incubator for $30 \mathrm{~min}$ to form a gel-like structure. The defined medium comprised DMEM/F12, 10\% knockout serum replacement (KSR) (Gibco), $2 \mu \mathrm{M}$ retinoic acid (RA) (Sigma), $100 \mathrm{ng} / \mathrm{ml}$ SCF (Peprotech), $100 \mathrm{ng} / \mathrm{ml}$ BMP4 (Peprotech), and $10^{-6} \mathrm{M}$ testosterone (Sigma). The defined medium was added to the gel slowly and changed every two days. The cells were cultured in $5 \% \mathrm{CO}_{2} / 95 \%$ air ( $\left.\mathrm{vol} / \mathrm{vol}\right)$ at $34{ }^{\circ} \mathrm{C}$ and classified three groups, namely $3 \mathrm{D}$-induced (3D-I) group (cells cultured in matrigel and defined medium), 3D-control (3D-C) group (human SSCs without Sertoli cells but with matrigel and defined factors), 3D-negative (3D-N) group (human SSCs with Sertoli cells and matrigel but without defined factors), 2D-induced (2D-I) group (cells without matrigel but with defined factors), and 2D-control (2D-C) group (cells without matrigel or defined factors). After induction for 5, 10, 15, and 20 days, cells from 3D-I were digested into single-cell suspension by disperse (Corning). According to the "Gold Standards" criteria for the in vitro-derived gametes, the cells were analyzed by various kinds of methods, including fluorescence-activated cell sorter (FACS), real-time PCR, immunocytochemistry, fluorescence in situ hybridization (FISH), multiplex realtime PCR, and RNA sequencing, to determine whether human spermatogenesis occurred in vitro.

\section{Flow cytometry analysis for DNA content}

Flow cytometry was performed to isolate $1 \mathrm{C}$ haploid cells and $4 \mathrm{C}$ cells and to evaluate differentiation efficiency of human SSCs by 3D-I and 2D-I system at day 20. Single-cell suspensions were prepared as described above. After being stained with $10 \mu \mathrm{g} / \mathrm{ml}$ Hoechst 33342 for $40 \mathrm{~min}$ at $34{ }^{\circ} \mathrm{C}$, 15,000 cells at different differentiation stages were analyzed and separated with a FACSAria system (Becton Dickinson).

\section{RNA isolation, RT-PCR, and real-time PCR}

Total RNA from human SSCs, the differentiated cells derived from human SSCs by 3D-I, 2D-I, 3D-C, 3D-N, and 2D-C, and human Sertoli cells was extracted with an RNeasy micro kit (QIAGEN). Reverse transcription (RT) was performed using a QuantiTect reverse transcription kit (QIAGEN). PCR and real-time (quantitative) PCR were performed with specific primers of selected genes (Suppementary Tabled S1 and S2). Quantitative PCR reactions were performed using Power SYBR ${ }^{\circledR}$ Green PCR Master Mix and a 7500 Fast Real-time PCR System (Applied 
Biosystems, Tokyo, Japan). To quantify the PCR products, we utilized the comparative $\mathrm{Ct}$ (threshold cycle) method described previously [47]. The threshold of cycle values was normalized against the threshold value of human housekeeping gene $A C T B$. All gene expression analyses were performed from three independent experiments.

\section{Immunocytochemistry}

Cells were fixed in 4\% PFA for $15 \mathrm{~min}$ at room temperature, permeabilized with $0.5 \%$ Triton X-100 for $30 \mathrm{~min}$, blocked with $5 \%$ BSA for $1 \mathrm{~h}$, and incubated with primary antibodies. The primary antibodies included GPR125 (Santa Cruz,1:200), GFRA1 (Santa Cruz,1:200), UCHL1 (AbD Serotec,1:200), PLZF (Abcam, 1:200), MAGEA4 (a kind gift from Professor Giulio C. Spagnoli, University Hospital of Basel, Switzerland, 1:25), SOX9 (Millipore, 1:200), WT1 (Santa Cruz, 1:200), SCF (Sigma, 1:200), BMP4 (Abcam, 1:200), PIWIL1 (Abcam, 1:200), PIWIL2 (Abcam, 1:200), $\gamma \mathrm{H} 2 \mathrm{AX}$ (Millipore, 1:100), Protamine 2 (PRM2, Santa Cruz, 1:200), Acrosin (ACR, Santa Cruz, 1:200), SYCP3 (Abcam, 1:100), H3K9 trimethylation (H3K9me3, Abcam, 1:200), and Human nuclear antigen (HumNuc, Millipore, 1:200). After incubation at $4{ }^{\circ} \mathrm{C}$ overnight, cells were washed three times in PBS (Sigma) and followed by incubation with secondary antibodies for 1 $\mathrm{h}$ at room temperature. Secondary antibodies were conjugated to Alexa Fluor 488 or Alexa Fluor 594 (Invitrogen). DAPI was used to label cell nuclei, and images were captured with a fluorescence microscope (Leica). Replacement of primary antibodies with isotype IgGs served as negative controls.

\section{Meiotic chromatin spread}

Meiotic chromatin spread assays were performed to determine the meiotic progression in human SSCs by 3D-I system according to the method described previously [24]. Cells were lysed by a hypotonic solution and spread evenly over slides layered with $1 \%$ PFA and $0.15 \%$ Triton X-100. Slides were dried for $24 \mathrm{~h}$ at room temperature in a humid chamber. Cells were treated with $0.04 \%$ photoflo for $5 \mathrm{~min}$ and blocked with $4 \%$ goat serum. Triple or duplicate immunostaining was performed in these cells using primary antibodies, including SYCP3 (Abcam, 1:100), CREST (Immunovision, 1:100), and MLH1 (Abcam, 1: 50), $\gamma \mathrm{H} 2 \mathrm{AX}$ (Abcam, $1: 100$ ), at $37^{\circ} \mathrm{C}$ for $2 \mathrm{~h}$ in a humid chamber. Goat anti-rabbit Alexa Fluor 594 (Invitrogen) and goat anti-human Alexa Fluor 488 secondary antibodies (Invitrogen) were applied at 1:200 dilution and incubated for $90 \mathrm{~min}$ at $37^{\circ} \mathrm{C}$. Cells were washed three times with PBS, and images were captured with a fluorescence microscope (Leica).

\section{Fluorescence in situ hybridization}

To establish chromosome counts in vitro-derived germ cells, a triple-color fluorescence in situ hybridization (FISH) was used to determined ploidy of these cells using centromeric probes against chromosomes 18, $\mathrm{X}$, and $\mathrm{Y}$ [48]. Briefly, round spermatids derived from human SSCs by 3DI system at day 20 , round spermatids from OA patients were separated by FACS, and human SSCs were isolated from OA patients by MACS. Cells were washed twice in PBS and fixed in freshly prepared Carnoy's fixative (3:1 for methanol: acetic acid) for $10 \mathrm{~min}$. One hundred microliters of cell suspension were placed into the center of unfrosted slides and they were treated with $1 \mathrm{mM}$ sodium hydroxide and saline-sodium citrate, respectively. Cells were dehydrated with series of graded alcohols, and $10 \mu \mathrm{l}$ mixtures of the probes $(18, \mathrm{X}, \mathrm{Y})$ were added to cells and sealed with rubber cement. Cell slides were placed into hybridization chamber and incubated at $78{ }^{\circ} \mathrm{C}$ for $8 \mathrm{~min}$ and $42^{\circ} \mathrm{C}$ overnight. The slides were transferred to the SSC/NP-40 washing solution at room temperature and washed for 3 min. DAPI was used to label cell nuclei, and images were captured with a fluorescence microscope (Leica). Replacement of chromosome probes with PBS was utilized as negative controls.

\section{Multiplex real-time PCR}

Multiplex real-time PCR was utilized to assess whether Y microdeletions existed in the round spermatids derived from human SSCs by 3D-I system and OA patients according to the manufacturer's instructions (Tellgen Corporation, Shanghai). Three different regions, namely AZFa, AZFb, and AZFc, were analyzed with six specific sequence-tagged site (STS) markers, including SY127, SY134, SY84, SY86, $S Y 254$, and $S Y 255$. Zinc finger protein, $X$-linked $(Z F X) /$ zinc finger protein, Y-linked $(Z F Y)$, and sex determining region $\mathrm{Y}(S R Y)$, were used as internal controls. DNA from normal human blood served as positive controls, and water without DNA but PCR with primers was used as negative controls.

\section{RNA sequencing}

To compare global gene expression, RNA sequencing analysis of round spermatids derived from human SSCs by 3D-I system, round spermatids of OA patients, and human SSCs and human Sertoli cells from OA patients was performed according to the procedure described previously [49]. Briefly, 20 round spermatids derived from human SSCs by 3D-I system, 20 round spermatids of OA patients, and 20 human SSCs and 20 human Sertoli cells from OA patients were isolated using micromanipulator, respectively. Round spermatids could be easily recognized by their small 
size and the presence of a round nucleus with a centrally located nucleolus. Round spermatids with acrosomic vesicle were selected, and total RNA was extracted from these cells using lysate buffer. The mRNA was enriched by Oligo(dT) and followed by mRNA fragment. The cDNA was synthesized by using random hexamer-primer and dNTPs, and size selection and PCR amplification were conducted. Agilent 2100 Bioanaylzer and ABI StepOnePlus real-time PCR system were used to qualify and quantify of the sample library. The library products were used for Illumina sequencing (Beijing Genomics Institute, Shenzhen, China). The data of RNA sequencing were verified with real-time PCR. The consistency between RNA sequencing data and real-time PCR was evaluated by Bland-Altman plot.

To compare global gene expression of the embryos derive from mouse oocytes fertilized with human round spermatids of human SSCs by 3D-I system and the embryos obtained from mouse oocytes fertilized with mouse round spermatids, RNA sequencing was conducted according to the procedure described above.

\section{Bisulfite sequencing}

Bisulfite sequencing was performed to compare genome DNA methylation of round spermatids derived from human SSCs by 3D-I system, normal round spermatids from OA patients, and human SSCs and pachytene spermatocytes of OA patients. Briefly, round spermatids derived from OA patients and from human SSCs by 3D-I system were separated by FACS and human pachytene spermatocytes were isolated from OA patients using STAPUT method, and DNA was extracted from these cells using the MicroElute genomic DNA kit (OMEGA). The quality and integrity of DNA of round spermatids derived from human SSCs by 3D-I system, normal round spermatids from OA patients, and human SSCs and pachytene spermatocytes of OA patients were assessed by the A260/A280 ration using the Nanodrop and agarose gel electrophoresis by Bioanalyzer 2100 (Agilen, Germany). For library preparation, 100ng of genomic DNA were digested by the methylation-insensitive restriction enzyme MspI to generate short fragments that contained $\mathrm{CpG}$ dinucleotide at the ends. DNA was endrepaired, A-tailed and ligated to methylated Illumina adapters utilizing the Truseq DNA Sample Prep Kit (Illumina, USA). Thereafter, the CpG-rich DNA fragments were subjected to bisulfite conversion using the MethylCode Bisulfite Conversion Gold Kit (Zymo, USA), and PCR reactions were amplified by uracil PCR ReadyMix (KAPA, USA). The libraries were sequenced on Illumina Hiseq $X$ with $2 \times 150$ bp paired-end sequencing, which was controlled by Hiseq Control Software (HCS). Bismark soft was used to analyze the methylation of H19 and PEG10 genes, and gene DNA methylation was analyzed when the $\mathrm{CpG}$ loci were covered by five reads.

\section{Round spermatid microinjection}

Microinjection of round spermatids (ROSI) was performed to evaluate the fertilization capacity of round spermatids derived from human SSCs 3D-I system pursuant to the procedure described previously [50]. Female B6D2F1 mice were superovulated by the injection of 5 IU equine chorionic gonadotropin and followed by 5 IU human chorionic gonadotropin (hCG) $48 \mathrm{~h}$ later. Cumulus-oocyte complexes were collected from oviducts at $14-16 \mathrm{~h}$ after hCG injection, and they were placed in Hepes-CZB medium and treated with $0.1 \%$ hyaluronidase to disperse cumulus cells. After being washed twice, oocytes were transferred to CZB medium to resume incubation. Human round spermatids were collected from fresh testicular cells of OA patients or from the cells derived from human SSCs by 3D-I system. Round spermatids could be easily recognized by their small size and the presence of a round nucleus with a centrally located nucleolus [51]. ROSI was performed using a micromanipulator with piezo-electric elements. The oocytes fertilized with human round spermatids from human SSCs by 3D-I system, human SSCs, and mouse round spermatids were incubated in $\mathrm{CZB}$ medium at $37{ }^{\circ} \mathrm{C}$ one and half hours and placed into $\mathrm{Ca}^{2+}$ free $\mathrm{CZB}$ containing $10 \mathrm{mM} \mathrm{SrCl}_{2}$ for $1 \mathrm{~h}$ to activate the oocytes. The oocytes fertilized with human round spermatids were incubated in CZB medium at $37{ }^{\circ} \mathrm{C}$ under $5 \% \mathrm{CO}_{2}$ in air to examine pronuclear formation and in vitro development. The experiments of ROSI was approved by The Institutional Ethical Review Committee of Ren Ji Hospital, and the hybrid embryos could be developed to no more than 8-cell stage and should be disrupted after RNA sequencing and $\mathrm{H} 3 \mathrm{~K} 9 \mathrm{~m} 3$ immunostaining analyses.

\section{Statistical analysis}

All data were presented as mean \pm SEM from at least three independent experiments. Statistical analyses were determined using Students $t$-test and $p<0.05$ was considered statistically significant.

Acknowledgements We greatly appreciate critical reading and revisions on this manuscript by Dr. C. Yan Cheng (Senior Scientist, Population Council, The Rockefeller University), Dr. Guang $\mathrm{Hu}$ (Senior Investigator, National Institute of Environmental Health Sciences, NIH), and Dr. Philip Jordan (Assistant Professor, Johns Hopkins University). We thank Professor Giulio C. Spagnoli, University Hospital of Basel, Switzerland, for providing the antibody to MAGEA4. This work was supported by key grants from National Nature Science Foundation of China (31230048) and Chinese Ministry of Science and Technology (2016YFC1000600, 2014CB943101), grants from National Science Foundation of China (31671550, 
31171422, 31401250), The Program for Professor of Special Appointment (Eastern Scholar) at Shanghai Institutions of Higher Learning (2012.53), Shanghai Municipal Education CommissionGaofeng Clinical Medicine Grant Support (20152511), Shanghai Hospital Development Center (SHDC12015122), and a key grant from the Science and Technology Commission of Shanghai Municipality (12JC1405900).

Author contributions Conceptualization, ZH and MS; methodology, QS; investigation, MS, QY, MN, HW, LW, CY, JH, ZC, HF, and FZ; human tissue collection, FZ and $\mathrm{ZL}$; writing, $\mathrm{ZH}$ and $\mathrm{MS}$, with all authors approving the final version; funding acquisition, $\mathrm{ZH}$, and QZ; supervision, ZH, ZL, SG, and WQG.

\section{Compliance with ethical standards}

Conflict of interest The authors declare that they have no competing interests.

Open Access This article is licensed under a Creative Commons Attribution-NonCommercial-ShareAlike 4.0 International License, which permits any non-commercial use, sharing, adaptation, distribution and reproduction in any medium or format, as long as you give appropriate credit to the original author(s) and the source, provide a link to the Creative Commons license, and indicate if changes were made. If you remix, transform, or build upon this article or a part thereof, you must distribute your contributions under the same license as the original. The images or other third party material in this article are included in the article's Creative Commons license, unless indicated otherwise in a credit line to the material. If material is not included in the article's Creative Commons license and your intended use is not permitted by statutory regulation or exceeds the permitted use, you will need to obtain permission directly from the copyright holder. To view a copy of this license, visit http://creativecommons. org/licenses/by-nc-sa/4.0/.

\section{References}

1. Hu Z, Xia Y, Guo X, Dai J, Li H, Hu H, et al. A genome-wide association study in Chinese men identifies three risk loci for nonobstructive azoospermia. Nat Genet. 2011;44:183-6.

2. Hu Z, Li Z, Yu J, Tong C, Lin Y, Guo X, et al. Association analysis identifies new risk loci for non-obstructive azoospermia in Chinese men. Nat Commun. 2014;5:3857.

3. Handel MA, Eppig JJ, Schimenti JC. Applying 'gold standards' to in vitro-derived germ cells. Cell. 2014;157:1257-61.

4. Hirsh A. Male subfertility. BMJ. 2003;327:669-72.

5. Wu B, Lu NX, Xia YK, Gu AH, Lu CC, Wang W, et al. A frequent $\mathrm{Y}$ chromosome $\mathrm{b} 2 / \mathrm{b} 3$ subdeletion shows strong association with male infertility in Han-Chinese population. Hum Reprod. 2007;22:1107-13.

6. De Kretser DM, Baker HW. Infertility in men: recent advances and continuing controversies. J Clin Endocrinol Metab. 1999;84:3443-50.

7. Saitou M, Miyauchi H. Gametogenesis from pluripotent stem cells. Cell Stem Cell. 2016;18:721-35.

8. Riboldi M, Rubio C, Pellicer A, Gil-Salom M, Simon C. In vitro production of haploid cells after coculture of CD49f + with Sertoli cells from testicular sperm extraction in nonobstructive azoospermic patients. Fertil Steril. 2012;98:580-90.e584.

9. Sousa M, Cremades N, Alves C, Silva J, Barros A. Developmental potential of human spermatogenic cells co-cultured with Sertoli cells. Hum Reprod. 2002;17:161-72.
10. Yang S, Ping P, Ma M, Li P, Tian R, Yang H, et al. Generation of haploid spermatids with fertilization and development capacity from human spermatogonial stem cells of cryptorchid patients. Stem Cell Rep. 2014;3:663-75.

11. Cremades N, Bernabeu R, Barros A, Sousa M. In-vitro maturation of round spermatids using co-culture on Vero cells. Hum Reprod. 1999;14:1287-93.

12. Hue D, Staub C, Perrard-Sapori MH, Weiss M, Nicolle JC, Vigier $\mathrm{M}$, et al. Meiotic differentiation of germinal cells in three-week cultures of whole cell population from rat seminiferous tubules. Biol Reprod. 1998;59:379-87.

13. Tanaka A, Nagayoshi M, Awata S, Mawatari Y, Tanaka I, Kusunoki H. Completion of meiosis in human primary spermatocytes through in vitro coculture with Vero cells. Fertil Steril. 2003;79 Suppl 1:795-801.

14. Tesarik J. Overcoming maturation arrest by in vitro spermatogenesis: search for the optimal culture system. Fertil Steril. 2004;81:1417-9.

15. Lee JH, Kim HJ, Kim H, Lee SJ, Gye MC. In vitro spermatogenesis by three-dimensional culture of rat testicular cells in collagen gel matrix. Biomaterials. 2006;27:2845-53.

16. Sato T, Katagiri K, Gohbara A, Inoue K, Ogonuki N, Ogura A, et al. In vitro production of functional sperm in cultured neonatal mouse testes. Nature. 2011;471:504-7.

17. Sato T, Katagiri K, Kubota Y, Ogawa T. In vitro sperm production from mouse spermatogonial stem cell lines using an organ culture method. Nat Protoc. 2013;8:2098-104.

18. Sato T, Katagiri K, Yokonishi T, Kubota Y, Inoue K, Ogonuki N, et al. In vitro production of fertile sperm from murine spermatogonial stem cell lines. Nat Commun. 2011;2:472.

19. Feng LX, Chen Y, Dettin L, Pera RA, Herr JC, Goldberg E, et al. Generation and in vitro differentiation of a spermatogonial cell line. Science. 2002;297:392-5.

20. Zhou Q, Wang M, Yuan Y, Wang X, Fu R, Wan H, et al. Complete meiosis from embryonic stem cell-derived germ cells in vitro. Cell Stem Cell. 2016;18:330-40.

21. He Z, Kokkinaki M, Jiang J, Dobrinski I, Dym M. Isolation, characterization, and culture of human spermatogonia. Biol Reprod. 2010;82:363-72.

22. West JA, Park IH, Daley GQ, Geijsen N. In vitro generation of germ cells from murine embryonic stem cells. Nat Protoc. 2006;1:2026-36.

23. Kee K, Angeles VT, Flores M, Nguyen HN, Reijo Pera RA. Human DAZL, DAZ and BOULE genes modulate primordial germ-cell and haploid gamete formation. Nature. 2009;462:222-5.

24. Panula S, Medrano JV, Kee K, Bergstrom R, Nguyen HN, Byers $B$, et al. Human germ cell differentiation from fetal- and adultderived induced pluripotent stem cells. Hum Mol Genet. 2011;20:752-62.

25. Holloway JK, Booth J, Edelmann W, McGowan CH, Cohen PE. MUS81 generates a subset of MLH1-MLH3-independent crossovers in mammalian meiosis. Plos Genet. 2008;4:e1000186.

26. Mahadevaiah SK, Turner JM, Baudat F, Rogakou EP, de Boer P, Blanco-Rodriguez J, et al. Recombinational DNA double-strand breaks in mice precede synapsis. Nat Genet. 2001;27:271-6.

27. Bland JM, Altman DG. Statistical methods for assessing agreement between two methods of clinical measurement. Lancet. 1986;1:307-10.

28. von Kopylow K, Kirchhoff C, Jezek D, Schulze W, Feig C, Primig $\mathrm{M}$, et al. Screening for biomarkers of spermatogonia within the human testis: a whole genome approach. Hum Reprod. 2010;25:1104-12.

29. Sasaki K, Yokobayashi S, Nakamura T, Okamoto I, Yabuta Y, Kurimoto K, et al. Robust in vitro induction of human germ cell fate from pluripotent stem cells. Cell Stem Cell. 2015;17:178-94. 
30. Irie N, Weinberger L, Tang WW, Kobayashi T, Viukov S, Manor YS, et al. SOX17 is a critical specifier of human primordial germ cell fate. Cell. 2015;160:253-68.

31. Hayashi K, Ohta H, Kurimoto K, Aramaki S, Saitou M. Reconstitution of the mouse germ cell specification pathway in culture by pluripotent stem cells. Cell. 2011;146:519-32.

32. Nakaki F, Hayashi K, Ohta H, Kurimoto K, Yabuta Y, Saitou M. Induction of mouse germ-cell fate by transcription factors in vitro. Nature. 2013;501:222-6.

33. Hikabe O, Hamazaki N, Nagamatsu G, Obata Y, Hirao Y, Hamada N, et al. Reconstitution in vitro of the entire cycle of the mouse female germ line. Nature. 2016;539:299-303.

34. Lee JH, Gye MC, Choi KW, Hong JY, Lee YB, Park DW, et al. In vitro differentiation of germ cells from nonobstructive azoospermic patients using three-dimensional culture in a collagen gel matrix. Fertil Steril. 2007;87:824-33.

35. Hou J, Niu M, Liu L, Zhu Z, Wang X, Sun M, et al. Establishment and characterization of human germline stem cell line with unlimited proliferation potentials and no tumor formation. Sci Rep. 2015;5:16922.

36. Stukenborg JB, Schlatt S, Simoni M, Yeung CH, Elhija MA, Luetjens CM, et al. New horizons for in vitro spermatogenesis? An update on novel three-dimensional culture systems as tools for meiotic and post-meiotic differentiation of testicular germ cells. Mol Hum Reprod. 2009;15:521-9.

37. Dores C, Dobrinski I. De novo morphogenesis of testis tissue: an improved bioassay to investigate the role of VEGF165 during testis formation. Reproduction. 2014;148:109-17.

38. Kita K, Watanabe T, Ohsaka K, Hayashi H, Kubota Y, Nagashima $\mathrm{Y}$, et al. Production of functional spermatids from mouse germline stem cells in ectopically reconstituted seminiferous tubules. Biol Reprod. 2007;76:211-7.

39. Watanabe T, Hayashi H, Kita K, Kubota Y, Ogawa T. Ectopic porcine spermatogenesis in murine subcutis: tissue grafting versus cell-injection methods. Asian J Androl. 2009;11:317-23.

40. Ungewitter EK, Yao HH. How to make a gonad: cellular mechanisms governing formation of the testes and ovaries. Sex Dev. 2013;7:7-20.
41. Pellegrini M, Filipponi D, Gori M, Barrios F, Lolicato F, Grimaldi $\mathrm{P}$, et al. ATRA and KL promote differentiation toward the meiotic program of male germ cells. Cell Cycle. 2008;7:3878-88.

42. Yang S, Bo J, Hu H, Guo X, Tian R, Sun C, et al. Derivation of male germ cells from induced pluripotent stem cells in vitro and in reconstituted seminiferous tubules. Cell Proliferat. 2012;45:91-100.

43. Carlomagno G, van Bragt MP, Korver CM, Repping S, de Rooij DG, van Pelt AM. BMP4-induced differentiation of a rat spermatogonial stem cell line causes changes in its cell adhesion properties. Biol Reprod. 2010;83:742-9.

44. Kawase E, Wong MD, Ding BC, Xie T. Gbb/Bmp signaling is essential for maintaining germline stem cells and for repressing bam transcription in the Drosophila testis. Development. 2004;131:1365-75.

45. Pellegrini M, Grimaldi P, Rossi P, Geremia R, Dolci S. Developmental expression of BMP4/ALK3/SMAD5 signaling pathway in the mouse testis: a potential role of BMP4 in spermatogonia differentiation. J Cell Sci. 2003;116:3363-72.

46. Rossi P, Dolci S. Paracrine mechanisms involved in the control of early stages of mammalian spermatogenesis. Front Endocrinol. $2013 ; 4: 181$.

47. He Z, Jiang J, Hofmann MC, Dym M. Gfra1 silencing in mouse spermatogonial stem cells results in their differentiation via the inactivation of RET tyrosine kinase. Biol Reprod. 2007;77:723-33.

48. Sarrate Z, Anton E. Fluorescence in situ hybridization (FISH) protocol in human sperm. J Vis Exp. 2009;31:1405.

49. Yan L, Yang M, Guo H, Yang L, Wu J, Li R, et al. Single-cell RNA-Seq profiling of human preimplantation embryos and embryonic stem cells. Nat Struct Biol. 2013;20:1131-9.

50. Li C, Mizutani E, Ono T, Wakayama T. An efficient method for generating transgenic mice using $\mathrm{NaOH}$-treated spermatozoa. Biol Reprod. 2010;82:331-40.

51. Yazawa H, Yanagida K, Sato A. Human round spermatids from azoospermic men exhibit oocyte-activation and $\mathrm{Ca}^{2+}$ oscillationinducing activities. Zygote. 2007;15:337-46. 